\title{
Biomaterial Approaches to Modulate Reactive Astroglial Response
}

\author{
Jonathan M. Zuidema ${ }^{a}$ Ryan J. Gilbert ${ }^{b, c}$ Manoj K. Gottipati ${ }^{b-d}$ \\ ${ }^{a}$ Department of Chemistry and Biochemistry, University of California, San Diego, CA, USA; ${ }^{b}$ Department of \\ Biomedical Engineering, Rensselaer Polytechnic Institute, Troy, NY, USA; ${ }^{C}$ Center for Biotechnology and \\ Interdisciplinary Studies, Rensselaer Polytechnic Institute, Troy, NY, USA; ${ }^{\mathrm{d} C e n t e r}$ for Brain and Spinal Cord \\ Repair, Department of Neuroscience, The Ohio State University, Columbus, OH, USA
}

\author{
Keywords \\ Astrocytes - Biomaterials · Spinal cord injury · Reactive \\ gliosis
}

\begin{abstract}
Over several decades, biomaterial scientists have developed materials to spur axonal regeneration and limit secondary injury and tested these materials within preclinical animal models. Rarely, though, are astrocytes examined comprehensively when biomaterials are placed into the injury site. Astrocytes support neuronal function in the central nervous system. Following an injury, astrocytes undergo reactive gliosis and create a glial scar. The astrocytic glial scar forms a dense barrier which restricts the extension of regenerating axons through the injury site. However, there are several beneficial effects of the glial scar, including helping to reform the blood-brain barrier, limiting the extent of secondary injury, and supporting the health of regenerating axons near the injury site. This review provides a brief introduction to the role of astrocytes in the spinal cord, discusses astrocyte phenotypic changes that occur following injury, and highlights studies that explored astrocyte changes in response to biomaterials tested within in vitro or in vivo envi-
\end{abstract}

ronments. Overall, we suggest that in order to improve biomaterial designs for spinal cord injury applications, investigators should more thoroughly consider the astrocyte response to such designs.

(c) 2018 S. Karger AG, Base

\section{Introduction}

Biomaterial development for repair and regeneration of the central nervous system (CNS) is an active area of investigation. Biomaterials can locally release therapeutics to the injury site to mitigate inflammation [Haggerty et al., 2018], provide neuroprotection [Haggerty and Oudega, 2013], and induce axonal regeneration [Tsintou et al., 2015]. Furthermore, scaffolds can be crafted to direct the extension of regenerating axons in the white matter tracts of the spinal cord [Straley et al., 2010; Schaub et al., 2016]. While to a large extent biomaterial development has focused on neuronal health and axonal regeneration, growing evidence suggests astrocytes play a pivotal role in supporting and inhibiting regeneration following spinal cord injury (SCI). Thus, future biomaterials crafted for the spinal cord will need to not only promote neuronal

\section{KARGER}

(c) 2018 S. Karger AG, Basel

E-Mail karger@karger.com

www.karger.com/cto
Manoj K. Gottipati, PhD

Department of Biomedical Engineering, Rensselaer Polytechnic Institute 110 8th Street

Troy, NY 12180 (USA)

E-Mail gottim2@ rpi.edu 
Table 1. Astrocyte physiological changes in response to injury

\begin{tabular}{|c|c|c|c|}
\hline Physiological marker & $\begin{array}{l}\text { Physiological change } \\
\text { following injury }\end{array}$ & Consequence of change & Reference \\
\hline $\begin{array}{l}\text { Glutamate transporter } \\
\text { expression }\end{array}$ & Expression decreases & $\begin{array}{l}\text { Increased extracellular } \\
\text { glutamate, leading to } \\
\text { excitotoxity }\end{array}$ & Olsen et al., 2010 \\
\hline \multirow[t]{2}{*}{ Connexin 43 expression } & Expression increases & $\begin{array}{l}\text { Increased } \mathrm{K}^{+} \text {uptake, reducing } \\
\mathrm{K}^{+} \text {excitotoxicity }\end{array}$ & Lee et al., 2005 \\
\hline & & $\begin{array}{l}\text { Increased } \mathrm{Ca}^{2+} \text { uptake, } \\
\text { improving } \mathrm{Ca}^{2+} \text { signaling } \\
\text { between astrocytes }\end{array}$ & Stout et al., 2002 \\
\hline Aquaporin expression & $\begin{array}{l}\text { Initially aquaporin expression } \\
\text { decreases; after } 2 \text { weeks, } \\
\text { aquaporin expression } \\
\text { increases }\end{array}$ & $\begin{array}{l}\text { Initial inability to sequester } \\
\text { water leads to tissue swelling }\end{array}$ & Nesic et al., 2006 \\
\hline Antioxidant production & $\begin{array}{l}\text { Antioxidant production } \\
\text { decreases }\end{array}$ & $\begin{array}{l}\text { Increased free radical damage to } \\
\text { spared tissue }\end{array}$ & Eddleston and Mucke, 1993 \\
\hline
\end{tabular}

health and axonal regeneration directly, but also shift astrocytes towards a phenotype more conducive to promoting neuronal survival and axonal regeneration through astrocyte-mediated mechanisms.

Since the prevailing focus of biomaterial design has emphasized the axon or neuron, few have developed biomaterials to modulate the astrocyte response and subsequently thoroughly assessed this response. If astrocytes are studied, then the quantification of the expression levels of the cytoskeletal marker glial fibrillary acidic protein (GFAP) [Hol and Pekny, 2015] is the most common parameter evaluated. Biomaterials that increase the expression of GFAP induce a more reactive astrocyte phenotype, which may not favorably support regeneration. Conversely, if the biomaterial reduces GFAP expression, then the biomaterial is thought to favorably support regeneration. However, in order to develop more advanced biomaterial designs, astrocyte assessment to implanted biomaterials must include an in-depth analysis rather than relying on analysis of a single cytoskeletal protein. As our understanding of astrocyte biology evolves, assessment of the astrocyte response should include a multifaceted array of different cytoskeletal proteins, growth factors, cytokines, extracellular matrix (ECM) molecules, ion transporters, and neurotransmitter channels as possible examples.

Biomaterial Modulation of Astrocytes
In this review, we focus on describing the normal behavior and physiology of astrocytes in uninjured tissue. Subsequently, we present a description of astrocyte phenotypic changes in response to injury. Then, the focus of the review shifts to a biomaterial-centered emphasis. We subsequently provide overviews of studies analyzing astrocyte response to biomaterials in culture. The review then analyzes those studies that place biomaterials into preclinical animal models of SCI and assess the astrocyte response to these biomaterials. Finally, we suggest those interested in developing biomaterials for SCI applications incorporate appropriate experiments to also assess the astrocyte response to such materials.

\section{Anatomical Structure/Function of Astrocytes in the Native, Uninjured CNS and Changes in Astrocyte Function following Injury}

Astrocyte anatomical structure, organization, and function has been very thoroughly reviewed in other publications [Kimelberg and Nedergaard, 2010; Sofroniew and Vinters, 2010]. In this review, we will focus this section on astrocyte parameters that would be important in designing biomaterials for the spinal cord. These parameters include astrocyte structure, function, and changes in 
astrocyte function following injury. The following sections present opportunity for biomaterial scientists to explore the ability of their design to influence or modulate specific astrocyte functions (Table 1).

\section{Anatomical Structure, Organization, and GFAP} Expression of Astrocytes in the Spinal Cord

Astrocytes are star-shaped glial cells that extensively populate the gray matter and white matter of the spinal cord, forming gap junctions between neighboring astrocytes [Scemes and Spray, 2012]. Glial cells outnumber neurons within the spinal cord with a glia-neuron ratio of 5.6 to 7.1 [Bahney and von Bartheld, 2018]. In the gray matter, astrocytes are protoplasmic, given this name since these astrocytes are large with several branched processes emanating from the center of the cell [Lundgaard et al., 2014] and weakly express GFAP [Miller and Raff, 1984; Hewett, 2009]. In the white matter, astrocytes are fibrous, consist of fewer processes than protoplasmic astrocytes, and orient and elongate along white matter tract axon bundles [Oberheim et al., 2012], where they are recognized through their prominent expression of GFAP [Eng et al., 2000]. When employing a biomaterial strategy for the spinal cord, it is important to note that assessment of GFAP expression in response to the biomaterial may not appreciably decipher differences in the fibrous and protoplasmic astrocyte response to the biomaterial.

\section{Astrocyte Regulation of Extracellular}

Neurotransmitter Glutamate Levels

Astrocytes interface with neurons at synapses where they control many aspects of synapse formation, function, and elimination [Chung et al., 2015]. Astrocytes uptake the excitatory amino acid glutamate released by neurons using two glutamate transporters: excitatory amino acid transporter 1 (EAAT1), also known as glutamate aspartate transporter (GLAST), and excitatory amino acid transporter 2 (EAAT2), which is known as glutamate transporter 1 (GLT-1) [Anderson and Swanson, 2000].

Fig. 1. Schematic showing the response of astrocytes to a SCI. NAIVE (top panel) shows astrocytes and microglia spread throughout an uninjured spinal cord. The astrocytes are predominantly present in the white matter and are organized in nonoverlapping domains. The inset on the right shows an individual astrocyte before injury. ACUTE (middle panel) shows an enhanced image of the acute changes observed following a contusion SCI. There is an activation of microglia, infiltrating macrophages, and astrocytes, all of which are recruited to the lesion site. The reactive astrocytes undergo hypertrophy and show an increase in intermediate fila-
Fibrous astrocytes, being more expressive of GFAP, also produce more glutamate transporters than protoplasmic astrocytes [Perego et al., 2000; Goursaud et al., 2009], suggesting that distinct astrocyte populations have specific functions. Since SCI induces loss of GLT-1 expression from astrocytes [Olsen et al., 2010], one may assess the success of a biomaterial to positively influence astrocytes through their capability to restore glutamate transporter production and glutamate uptake.

\section{Astrocyte Regulation of Extracellular/Intracellular Ion Concentrations}

Astrocytes are known to buffer extracellular $\mathrm{K}^{+}$ion concentrations at the synapse through the use of an astrocyte-specific connexin, connexin $43(\mathrm{Cx} 43)$ [Nagy and Rash, 2000]. Cx43 expression in the spinal cord occurs in white matter or fibrous astrocytes, but are also expressed in the fine processes of astrocytes globally [Ochalski et al., 1996]. Following SCI, Cx43 expression from gray matter astrocytes is increased 3-fold 4 weeks postinjury [Lee et al., 2005], possibly to handle the increased extracellular $\mathrm{K}^{+}$concentrations observed following SCI [Young and Koreh, 1986]. Thus, a biomaterial strategy that induces astrocytes to produce $\mathrm{Cx} 43$ or sequester $\mathrm{K}^{+}$following acute injury may improve outcomes following SCI.

Neurons communicate directly with astrocytes through the binding of neurotransmitters onto the surfaces of astrocytes, sequestering $\mathrm{Ca}^{2+}$ from astrocytic intracellular stores [Agulhon et al., 2008]. Also, astrocytes communicate intercellularly with nearby astrocytes through calcium waves that pass through $\mathrm{Cx} 43$ [Stout et al., 2002]. Following injury to the CNS, adenosine triphosphate (ATP) release triggers astrocytes to increase their cytoplasmic calcium concentrations [Burda et al., 2016]. Thus, examining how biomaterials influence $\mathrm{Ca}^{2+}$ dynamics in astrocytes may lead to the design of substrates supporting astrocyte phenotypes supportive of regeneration or repair. $\mathrm{Ca}^{2+}$ dynamics in astrocytes is not often studied with biomaterials. However, microgroove

ment (IF) expression, proliferation, and neurotoxin release, changes presented in an enlarged inset on the right along with the associated text. There is also a significant axonal dieback and an accumulation of myelin debris. CHRONIC (bottom panel) shows the chronic changes observed following a contusion SCI. The reactive astrocytes increase their production of proteoglycans and form a thick glial scar, as presented in the enlarged inset on the right with the text. The activated microglia and macrophages also persist within the lesion area. 


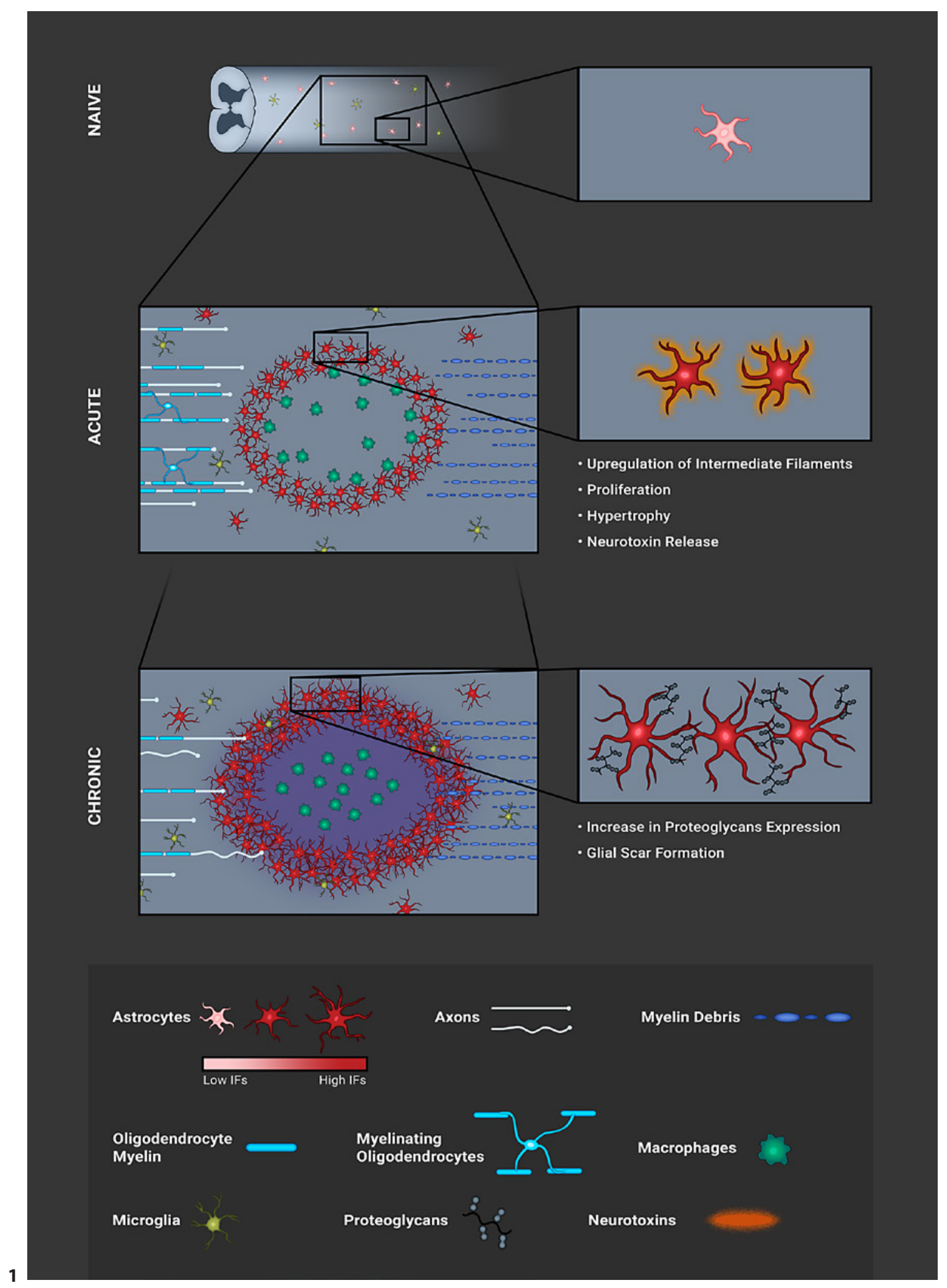


substrates induced astrocytes to increase ATP exocytosismediated calcium signaling compared to astrocytes cultured on flat surfaces [Singh et al., 2015].

Astrocytes also help control extracellular $\mathrm{pH}$ through transporting enzymes for $\mathrm{H}^{+}$and $\mathrm{HCO}_{3}{ }^{-}$[Kimelberg and Nedergaard, 2010]. Fibrous [Hansen et al., 2015] and protoplasmic [Dela Peña et al., 2014] astrocytes have the capability of regulating extracellular $\mathrm{pH}$. Injury to the CNS reduces extracellular $\mathrm{pH}$ [Chesler, 2005], leading to astrocyte death by acidosis. Thus, a biomaterial approach that sequesters $\mathrm{H}^{+}$following acute SCI may lead to the rescuing of astrocytes, thereby leading to better outcomes following SCI.

\section{Astrocyte Regulation of Water Transport}

Astrocytes produce water transporters known as aquaporins which reside around perivascular end-feet at the blood-brain barrier [Kimelberg and Nedergaard, 2010]. Astrocytes predominantly express aquaporin 4 (AQP4) which enable astrocytes to regulate water homeostasis quickly in the space-confined CNS [Potokar et al., 2016]. Following SCI at the thoracic region of male rats, astrocytes initially decreased AQP4 expression, but after 2 weeks astrocytes increased and maintained this increased expression of AQP4 in the chronically injured spinal cord [Nesic et al., 2006]. Since AQP4 expression by astrocytes in response to a biomaterial is understudied, a biomaterial scientist may explore the ability of their biomaterial design to regulate AQP4 expression and water uptake by astrocytes.

\section{Astrocyte Control of Blood Flow}

Astrocytes physically interact with blood vessels and can alter blood vessel diameter and blood flow through the expression of chemical mediators [Sofroniew and Vinters, 2010]. Global ischemia is known to increase nitric oxide synthase production from reactive astrocytes [Endoh et al., 1994] in an attempt to decrease local blood pressure. Release of ATP by damaged tissue stimulates astrocytes to rapidly release arachidonic acid and prostaglandin E2 [Xia and Zhu, 2011] to dilate nearby blood vessels. Thus, assessment of a biomaterial's capability to trigger an astrocyte to release vasodilators may predict the ability of the biomaterial to help reduce blood loss following acute injury.

\section{Astrocyte Antioxidant Capability}

Astrocytes produce several antioxidants capable of counteracting oxidative stress [Wilson, 1997]. Following SCI, the concentrations of many free radicals increase
[Visavadiya et al., 2016]. Thus, one approach to counteract free radical-induced secondary injury is to inject antioxidants near the injury site or systemically [Hall, 2011]. As the CNS ages [Clarke et al., 2018] or through the onset of injury [Eddleston and Mucke, 1993], astrocyte production of antioxidant decreases. Therefore, biomaterial scientists should examine the ability of the biomaterial to mediate astrocyte production of antioxidants.

\section{Summary of $\mathrm{SCl}$ and Ensuing Change of Astrocytes into a Reactive Phenotype}

While the previous sections have focused on specific functions of astrocytes and how injury changes astrocyte function, in the following sections a brief description of SCI globally is presented. Furthermore, astrocyte changes into reactive phenotypes is presented (Fig. 1). These sections are to inform biomaterial scientists of the possibilities of assessing if a biomaterial approach may influence astrocyte reactivity. Following a penetrating force or a blunt force trauma to the spinal cord, this primary injury damages and destroys cells at the site of impact. After initial cell damage/death, a cascade of biological processes collectively referred to as the "secondary injury" ensues which begins minutes after the initial insult and can continue for weeks [DeVivo et al., 2002; Cramer et al., 2005; Yiu and He, 2006]. Secondary injury is complex and involves several different biological processes described in more detail here. In the acute phase of the secondary injury, the blood-brain barrier is compromised, and bloodborn inflammatory cells infiltrate the injury site while resident microglia become activated. Injury compromises the blood vasculature, including hemorrhage, vasospasm, and thrombosis, leading to tissue edema, necrosis, and ischemia [Tator, 1991; Tator and Fehlings, 1991; Bareyre and Schwab, 2003]. Neural death at the injury site induces the release of glutamate, leading to an increase in the extracellular glutamate concentration. High extracellular glutamate concentrations can overstimulate neuronal glutamate receptors, leading to additional neuronal death [Farooque et al., 1996; Xu et al., 1998; McAdoo et al., 1999]. Injury also disrupts the ionic balance as well as free radical formation, which also results in neural death [Hall and Braughler, 1982; Stys, 1998]. Injury also induces apoptosis cascades in neurons, oligodendrocytes, and microglia [Beattie et al., 2000]. Infiltrating leukocytes release cytokines and reactive oxygen species, leading to further tissue damage [Means and Anderson, 1983; Popovich et al., 1997; Taoka et al., 1997; Mabon et al., 2000]. 
The inflammation also contributes to demyelination and the damaged white matter axons undergo dieback from the site of the injury, which progresses over long periods [Totoiu and Keirstead, 2005; Horn et al., 2008; Almad et al., 2011]. The accumulating myelin debris containing various inhibitory molecules inhibits axonal regeneration and acts as a potent inflammatory stimulus causing further spinal cord damage [McKerracher et al., 1994; Chen et al., 2000]. Although these multicellular inflammatory responses lead to tissue loss, they also serve several important functions, such as preventing infection, clearing cellular debris, and containing and preventing the spread of tissue damage. In addition, secondary injury cascades can also foster neural tissue repair [Donnelly and Popovich, 2008].

In the chronic phase, there is an increase in the connective tissue deposition and reactive gliosis, leading to the formation of a glial scar [Katoh-Semba et al., 1995]. The scar primarily consists of reactive astrocyte mixed with microglia and infiltrating macrophages. The axons with limited regenerating potential reach the vicinity of the injury site and struggle into the lesion penumbra, but eventually cease extending and become dystrophic as they penetrate deep into the glial scar [Davies et al., 1999; Grimpe, 2004]. Thus, the glial scar acts as a barrier for regenerating axons and hinders the possibility of functional recovery [Cregg et al., 2014].

Here, we highlight the changes of astrocytes from the time of injury to the formation of the glial scar. After SCI, astrocytes are activated in a process known as "reactive astrogliosis," where the astrocytes undergo a spectrum of changes in their gene expression, proliferation, morphology, and physiology [Anderson et al., 2014]. The astrocytes show pronounced hypertrophy and extend their processes to overlap with nearby astrocytes [Sofroniew and Vinters, 2010]. This overlapping of processes is only observed with fibrous astrocytes with very minimal overlap reported with protoplasmic astrocytes [Wilhelmsson et al., 2006; Sun et al., 2010].

Concomitant with the changes in morphology, reactive astrocytes increase their production of the intermediate filaments, namely GFAP and vimentin, which are the widely used histological markers for reactive astrocytes [Bignami and Dahl, 1974; Barrett et al., 1981; Eng, 1985; Yang et al., 1994]. These changes are also associated with a modest increase in the proliferation of the astrocytes around the lesion core [Faulkner, 2004]. Reactive astrocytes also secrete potent molecules such as lipocalin, which is known to promote neuronal death [Rathore et al., 2011; Bi et al., 2013]. Reactive astrocytes also express

Biomaterial Modulation of Astrocytes a range of inhibitory ECM proteins known as proteoglycans. The proteoglycans are a family of molecules constructed from a protein core with long glycosaminoglycan (GAG) chains consisting of repeating disaccharide units [Johnson-Green et al., 1991]. Astrocytes produce four classes of proteoglycans: chondroitin sulfate proteoglycans (CSPGs), keratin sulfate proteoglycans, heparin sulfate proteoglycans, and dermatan sulfate proteoglycans. Unique proteoglycans have specific protein cores and a variable number, length, and pattern of sulfation on the GAG side chains. Of these, CSPGs are prominent in the CNS and many distinct molecules exist, including aggrecan, brevican, neurocan, NG2, phosphacan, and versican [Dyck and Karimi-Abdolrezaee, 2015]. After injury, proteoglycan expression is rapidly upregulated by reactive astrocytes, forming an inhibitory gradient that is highest at the lesion center and diminishes gradually into the penumbra [Yiu and He, 2006]. The proteoglycans form the ECM for the glial scar and house reactive astrocytes, microglia, infiltrating macrophages, and endothelial cells [Jin and Yamashita, 2016].

Although the process of astrogliosis and the formation of the glial scar are well understood, the mechanisms underlying this process had not been well characterized until recently. It was shown that an insult to the spinal cord transforms the native astrocytes into reactive astrocytes, which eventually become scar-forming astrocytes [Hara et al., 2017]. All these distinct astrocytic phenotypes have distinct gene expression profiles associated with them. It was also reported that the astrocytic fate is dependent on environmental cues and the formation of astrocytic scar is driven by type 1 collagen via the integrin- $\mathrm{N}$-cadherin pathway.

An in-depth genomic analysis of reactive astrocytes has shown that reactive astrocytes exist in two distinct reactive states, one being detrimental (A1) and the other being neuroprotective (A2) depending on the nature of the insult [Zamanian et al., 2012; Liddelow and Barres, 2017]. A1 reactive astrocytes were observed when mice were exposed to a neuroinflammatory agent while astrocytes isolated from an ischemia-induced insult triggered astrocytes to become $\mathrm{A} 2$ reactive astrocytes. A follow-on study has recently revealed that the resident microglia activated by the inflammatory stimulus drives this A1 reactive state in astrocytes [Liddelow et al., 2017].

It was long hypothesized that the removal of the barricade created by astrocytes is the key to axonal regeneration following SCI. However, there is increasing evidence to show that astrogliosis is a physiological response required to restoring the internal homeostasis of the CNS during the 
acute phase of injury. Astrogliosis restores the blood-brain barrier and separates healthy tissue from the necrotic primary lesion. This separation also prevents the potential exacerbation of the inflammatory response, cellular death, and tissue damage occurring during the secondary injury [Bush et al., 1999; Faulkner, 2004; Okada et al., 2006; Herrmann et al., 2008]. The beneficial characteristics of astrogliosis was recently reported where the astrocyte scar actually aids in axonal regeneration rather than inhibiting it [Anderson et al., 2016; Liddelow and Barres, 2016].

\section{Analysis of Astrocyte in vitro Response to Biomaterials}

As the postinjury astrocyte response has become recognized as an important contributor to functional recovery following traumatic SCI [Silver and Miller, 2004; Anderson et al., 2016], researchers have explored how astrocytes interact with tissue engineering biomaterials. Hydrogels, polymer scaffolds, patterned substrates and surfaces, and other innovative biomaterial technologies have been developed to induce spinal cord regeneration and improve functional outcomes after injury [Gilbert et al., 2011]. In order to design biomaterials that favorably interact with astrocytes following SCI, in vitro experiments are used to understand the response of astrocytes to the presence of different biomaterials and surfaces. These studies determine astrocyte adhesion, morphological changes (Fig. 2), proliferation, migration, gene expression, and protein expression in an effort to engineer new biomaterial technologies that will more favorably interface with astrocytes following SCI.

\section{Astrocyte in vitro Response to Hydrogel Biomaterials}

Hydrogels are hydrophilic polymer networks that are commonly used in regenerative medicine to provide a permissive extracellular growth environment following injury, deliver cell-based therapies, and/or release therapeutics for extended time periods [for a comprehensive

Fig. 2. Astrocyte morphologies imaged in vitro. Astrocytes demonstrate several morphologies in vitro depending on their culture environment. (1) On typical smooth, 2D surfaces astrocytes demonstrate a flat, spread morphology. (2) Astrocytes cultured in 3D hydrogels show many different morphologies, including stellate astrocytes that extend processes in all directions, bipolar astrocytes that extend two processes, round astrocytes with no processes, and perivascular astrocytes that extend processes that resemble endfeet. (3) Astrocytes cultured on isotropic microtopographies pre- review of hydrogel design for regenerative medicine, see Annabi et al., 2014]. The capability to inject most biologically relevant hydrogels directly into an injury site and have them conform to the lesion geometry, as well as the ability to engineer hydrogel mechanical properties, degradation times, and drug release characteristics, make these biomaterials attractive for regenerative medicine. These advantageous properties of hydrogels have led to their use as biomaterial therapeutics to improve recovery following SCI [Jain et al., 2006; Dumont et al., 2016]. In order to more fully understand how astrocytes interact with hydrogels, in vitro experiments are used to study their response (Table 2).

Astrocytes cultured on two-dimensional (2D) cover glass or tissue culture plastic display a spread morphology that closely resemble the morphology of reactive, in vivo astrocytes found boarding the lesion [Zamanian et al., 2012]. The three-dimensional (3D) environment presented by hydrogels makes them especially attractive, since astrocytes can grow in a $3 \mathrm{D}$ environment that is similar to their in vivo milieu. In fact, it was observed that primary rat cortical astrocytes cultured in $3 \mathrm{D}$ collagen type 1 hydrogels are less reactive than those cultured on 2D surfaces [East et al., 2009]. In these studies, a reduction of reactive marker immunofluorescence (GFAP, CSPGs, and AQP4) was observed when astrocytes were cultured in the 3D collagen hydrogels. The authors also observed a reduction in mRNA levels of GFAP and neurocan. Similarly, astrocytes encapsulated in 3D alginate hydrogels exhibit stellate morphologies and extensive processes outgrowth [Frampton et al., 2011].

$3 \mathrm{D}$ cultures can be used to more accurately model the in vivo environment. 3D collagen hydrogels seeded with the C8 D1A astrocyte cell line have been used to study brain endothelial barrier function in vitro. Stimulation of C8 D1A cells cultured in 3D collagen hydrogels with transforming growth factor (TGF) $-\beta_{1}$ had a more pronounced effect on transendothelial electrical resistance compared to 2D monolayers of C8 D1A cells [Hawkins et al., 2015]. 3D cultures of rat cortical astrocytes seeded in collagen hydro-

dominately display stellate morphologies, with extension of several processes. (4) Astrocytes cultured on anisotropic micropatterns and microstructures display both bipolar and stellate morphologies that align along the direction of the surface they are cultured on. The many different morphologies suggest that astrocytes are impacted by their culture environment, and further study is needed to gain a full understanding of the astrocyte response to biomaterials.

(For figure see next page.) 
gels can also be used to test the reactivity response of astrocytes to potential stem cell therapies. Neural crest stem cells and differentiated adipose-derived stem cells cultured on top of collagen hydrogels seeded with astrocytes showed no increase in astrocyte reactivity, while bone marrow mesenchymal stem cells and Schwann cells caused reactivity in 3D cultured astrocytes [East et al., 2013]. Since astrocytes are not as reactive in 3D and appear to more accurately mimic a healthy tissue phenotype, others have studied how astrocytes impact neurite outgrowth. Cortical rat

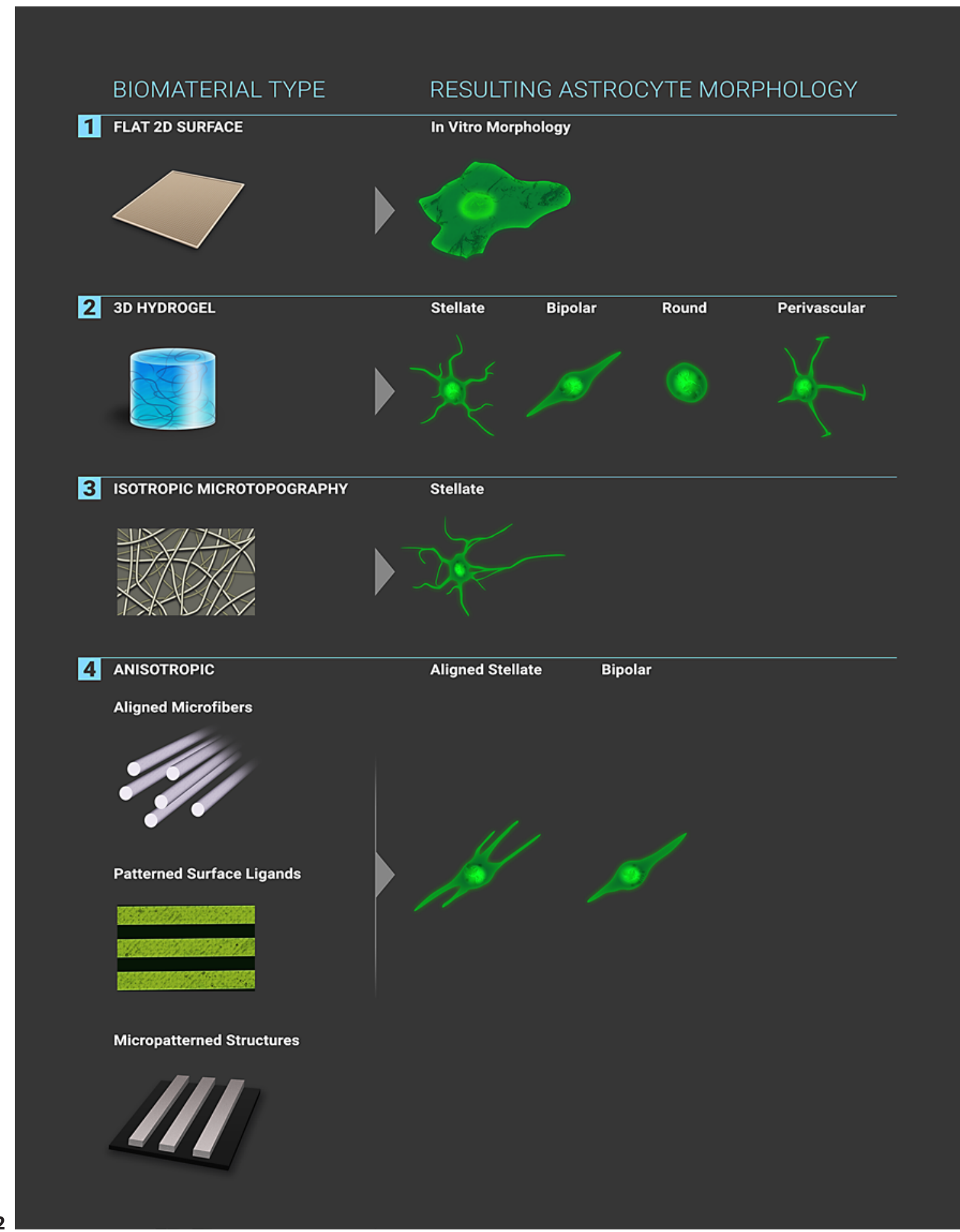


Table 2. In vitro response of astrocytes to hydrogels

\begin{tabular}{|c|c|c|c|}
\hline Type of hydrogel & Type of astrocytes & Astrocytic changes & Reference \\
\hline Collagen type 1 & Rat cortical & Reduced reactivity markers & East et al., 2009 \\
\hline Collagen type 1 & C8 D1A & Altered transendothelial electrical resistance & Hawkins et al., 2015 \\
\hline Collagen type 1 & Rat cortical & $\begin{array}{l}\text { Changes in reactivity induced by various cell } \\
\text { types }\end{array}$ & East et al., 2013 \\
\hline Alginate & Rat cortical & Conditioned medium increased reactivity & Rocha et al., 2015 \\
\hline Collagen type 1 & Mouse cortical & In vivo-like morphologies & Balasubramanian et al., 2016 \\
\hline Agarose/collagen type 1 & Rat cortical & Aligned astrocyte growth & Winter et al., 2016 \\
\hline Collagen type 1 & Rat cortical & FGF-2-induced migration & Macaya et al., 2013 \\
\hline $\begin{array}{l}\text { Collagen type } 1 / \text { hyaluronic } \\
\text { acid/Matrigel }\end{array}$ & Human derived & $\begin{array}{l}\text { Reduced GFAP expression in composite } \\
\text { hydrogels }\end{array}$ & Placone et al., 2015 \\
\hline Collagen type 1 & Rat midbrain & $\begin{array}{l}\text { Micromotion increased perimeter and cell } \\
\text { area }\end{array}$ & Spencer et al., 2017 \\
\hline Collagen type 1 & DI TNC1 & Compression induced reactivity & Mulvihill et al., 2018 \\
\hline
\end{tabular}

astrocytes and cortical neurons cultured in 3D alginate hydrogels with fibroblast-conditioned medium induced a reactive astrocyte phenotype, altering their gene and protein expression towards a more reactive state while inhibiting neurite outgrowth [Rocha et al., 2015].

Balasubramanian et al. [2016] conducted an in-depth study of the morphological ontogeny of P1-P3 mouse cortical astrocytes cultured in 3D rat tail collagen type 1 hydrogels. Astrocytes grown in 3D collagen hydrogels exhibited bipolar and stellate morphologies, while these morphologies were rarely visualized following 4 days of 2D astrocyte monolayer culture. They also observed astrocytes with a perivascular morphology that showed structures resembling end-feet after 10 days of 3D culture, while this morphology was never observed in 2D. These results suggest that astrocytes cultured in $3 \mathrm{D}$ collagen hydrogels more closely approximate normal ontogeny. Tubular hydrogel-collagen microcolumns have been used to induce an aligned, bipolar morphology in rat cortical astrocytes seeded in these constructs [Winter et al., 2016]. The astrocytes growing in these constructs direct neurite extension along their axis of growth when cortical neurons were coseeded in the scaffolds.

Astrocyte migration into 3D hydrogels following SCI is important for neuron survival past the edge of the le- sion site. In order to study this phenomenon in vitro prior to implantation, rat cortical astrocytes were seeded into a "core" 3D collagen type 1 hydrogel [Macaya et al., 2013]. An encapsulating collagen hydrogel was then added around the core astrocyte gel, with fibroblast growth factor-2 (FGF-2) incorporated into these hydrogels. When FGF-2 was sequestered in the hydrogel or loaded into liposomes, astrocytes migrated farther into the encapsulating hydrogels. Overall, these studies suggest that the use of $3 \mathrm{D}$ hydrogels creates an in vitro environment that more closely mimics the in vitro milieu of astrocytes compared to 2D monolayer cultures.

The mechanical properties of hydrogels play an important role in the astrocyte response to these biomaterials [Georges et al., 2006]. Polyacrylamide and fibrin hydrogels fabricated with a shear modulus similar to that of tested rat brains demonstrated preferential growth to cultured cortical neurons, while astrocytes had round morphologies and disorganized actin filaments when cultured on the hydrogels [Georges et al., 2006]. Similarly, astrocytes cultured on soft hydrogels composed of chitosan, alginate, and genipin showed a round morphology as opposed to the typical 2D morphology of astrocytes cultured on tissue culture plastic or cover glass [McKay et al., 2014]. Placone et al. [2015] conducted a very elegant 
Table 3. In vitro response of astrocytes to biomaterial surface modifications

\begin{tabular}{|c|c|c|c|}
\hline Surface modification & Type of astrocytes & Astrocytic changes & Reference \\
\hline Hydrophobic polymers & Rat cortical & $\begin{array}{l}\text { Decreased adhesion with increased } \\
\text { hydrophobicity }\end{array}$ & Biran et al., 1999 \\
\hline Fractal tripalmitin & Rat cortical & $\begin{array}{l}\text { Morphology and protein expression } \\
\text { patterns closer to in vivo phenotypes }\end{array}$ & Hu et al., 2014 \\
\hline Printed laminin lanes & Rat cortical & Aligned astrocyte growth & Meng et al., 2012 \\
\hline Fibrinogen coated & Rat cortical & Fibrinogen removal, expression of CSPGs & Hsiao et al., 2013 \\
\hline Patterned collagen type 1 hydrogels & Rat cortical & Aligned growth, altered CSPG production & Hsiao et al., 2015 \\
\hline Aggrecan-laminin gradients & Rat cortical & Astrocytes migrate away from aggrecan & Hsiao et al., 2018 \\
\hline
\end{tabular}

study outlining the impact that hydrogel composition and mechanical properties have on the state of human astrocyte reactivity. They found that while most in vitro studies of astrocytes cultured in 3D hydrogels use collagen as their matrix, astrocytes cultured in composite hydrogels composed of collagen, hyaluronic acid (HA), and Matrigel exhibited similar morphological characteristics to those in the human brain and had very low levels of GFAP expression compared with astrocytes seeded in collagen hydrogels. This impactful study demonstrated the necessity for careful design of hydrogel systems to induce desirable astrocyte interactions with the 3D biomaterial environment.

Movement-induced micromotion of implanted hydrogels is another important mechanical property that can impact cellular responses in a 3D environment [Gilletti and Muthuswamy, 2006]. Therefore, the astrocyte response to linear actuator-induced micromotion was studied in 3D collagen type 1 hydrogels [Spencer et al., 2017]. Astrocytes responded to the micromotion by increasing their overall perimeters and total cell areas. Pathological deformation is another cause of the astrocyte transition from quiescent to reactive state [Hernandez, 2000], and compression forces can be used in vitro to study astrocyte mechanobiology. DI TNC1 astrocytes were seeded in a 3D rat tail collagen type 1 gel, and compressive loading was used to induce astrocyte reactivity [Mulvihill et al., 2018]. This in vitro model is an early step towards a means of developing a better understanding of how astrocytes respond to compressive forces.

Hydrogels provide a growth permissive, 3D environment that more closely resembles the in vivo astrocyte environment. They more appropriately model the in vivo astrocyte milieu compared with monolayer cultures of astrocytes and keep astrocyte in a more quiescent state than astrocytes cultured on 2D surfaces. However, as Placone et al. [2015] demonstrated, care must be taken to appropriately design a hydrogel that interfaces with astrocytes, especially since recent findings demonstrated that reactive astrocytes induce formation of the astrocytic scar when they interact with type 1 collagen [Hara et al., 2017]. Ongoing research in the field is working toward engineering hydrogels that will promote astrocytes to create a 3D growth environment that is conducive to neuron regeneration.

\section{Astrocyte in vitro Response to Ligand Patterned Surfaces}

It has long been established that ECM components impact the function of astrocytes [Nagano et al., 1993], and following SCI the composition of the ECM is altered to an axon inhibitory environment [Silver and Miller, 2004]. This has led researchers to study how surface chemistry and surface patterning with ECM components impact astrocyte growth in vitro (Table 3 ). The laboratories of Tresco and Hlady have been at the forefront of this field 
[Biran et al., 1999; Meng et al., 2012; Hsiao et al., 2013, $2015,2018]$. The importance of surface chemistry on astrocyte growth was one of the first variables studied. Initially, rat cortical astrocyte adhesion decreased with increasing hydrophobicity [Biran et al., 1999], while DNA synthesis increased with increasing hydrophobicity. However, all materials tested, except for poly-2-hydroxyethyl methacrylate, were capable of supporting astrocyte adhesion and growth to confluence within $2-5$ days. Water-repellant fractal tripalmitin surfaces were used to understand how astrocytes respond to surfaces with water contact angles of $150-160^{\circ}$. It was found that astrocytes cultured on these surfaces have both morphologies and protein expression patterns that are similar to in vivo phenotypes compared with flat poly-L-lysine controls [Hu et al., 2014]. Chitosan-, gelatin-, and poly-L-lysinebased films have also been studied to determine astrocyte growth on surfaces of differing compositions, and it was shown that chitosan and gelatin films supported greater astrocyte viability and proliferation compared with the other films tested [Martín-López et al., 2012].

Patterning of glass surfaces with ECM molecules has been used to study the impact of ECM on astrocyte growth. Microcontact printing consisting of $15-\mu \mathrm{m}$-wide laminin lanes was used to induce alignment of cultured rat cortical astrocytes [Meng et al., 2012]. Astrocytes aligned parallel to the laminin lanes and were able to direct neurite extension due to the alignment of their expressed fibronectin. Fibrinogen has also been adsorbed to glass surfaces in order to study how rat cortical astrocytes may interact with blood on a biomaterial surface. Hsiao et al. [2013] found that astrocytes remove fibrinogen and express CSPGs at the loci of fibrinogen stimuli. This response was found to be independent of TGF $\beta$ and suggests that implant-adsorbed fibrinogen may contribute to reactive astrogliosis. Surface patterning of biomaterials has the potential to induce cellular alignment, so astrocytes were studied on ECM-patterned collagen hydrogels [Hsiao et al., 2015]. Patterning of the collagen hydrogels with fibrinogen, aggrecan, fibronectin, and laminin induced aligned growth of astrocytes, while patterns of fibrinogen, aggrecan, and laminin lowered CSPG expression visualized using immunocytochemistry.

Aggrecan-laminin dot gradients were developed to study how astrocytes respond to molecular cues of the postinjury environment. These gradients were fabricated using microcontact printing, where uniformly coated laminin covered the entire surface while micrometersized dots of aggrecan were randomly positioned [Hsiao et al., 2018]. The study found that astrocytes preferen- tially shifted away from aggrecan areas to areas with higher laminin surface coverage. Microcontact printing is also a useful tool to study small numbers in a constricted growth region. This technology has been applied to astrocyte-neuron cocultures, where astrocytes are seeded onto isolated rings of ECM proteins separated by surface-adsorbed polyethylene glycol (PEG) [Ricoult et al., 2012]. Hippocampal neurons were then seeded on the "microisland" cultures of astrocytes, demonstrating that these cocultures can be used to study the cellular response to different surface adsorbed extracellular proteins. This "microisland" culture platform was further improved upon by using parylene- $\mathrm{C} / \mathrm{SiO}_{2}$ surfaces to selectively bond PEG to $\mathrm{SiO}_{2}$ surfaces [Raos et al., 2018]. Surface immobilization of proteins by covalent attachment is another method that can be used to study the response of astrocytes to different surface patters. Covalent attachment of the L1 biomolecule and fibronectin to silicon surfaces was used to show that astrocytes prefer to adhere to surfaces with attached fibronectin compared to L1 [Azemi et al., 2008].

The ECM environment following SCI has a very complex geometry, shows temporal changes, and is composed of many different ECM molecules. Because of this complexity, surface patterning is a useful in vitro technique to elucidate the influence that single and/or multiple ECM molecules have on astrocyte growth and response. This technology can be used going forward for the in-depth study of how known ECM molecule concentrations, gradients, spatial configurations, and temporal changes impact astrocytes. This area of research has the potential to lead to a greater understanding of how the in vivo ECM environment following SCI alters the astrocyte response.

\section{Astrocyte in vitro Response to Surface Topography}

Cells sense the micro- and nanotopography of their extracellular environment, leading to the design of surfaces that alter cellular responses [Ross et al., 2012], and, following SCI, astrocytes near the injury site undergo reactive astrogliosis in response to change in their extracellular environment [Hara et al., 2017]. Therefore, many studies have investigated the interplay between surface topography and astrocyte response to such topographies (Table 4). Aligned micro- and nanostructures made using imprint lithography [Xie and Luttge, 2014], microcontact printing [Recknor et al., 2004], mechanical collagen-fiber alignment [Chaubaroux et al., 2015], parylene-C patterning [Unsworth et al., 2011], and aligned polymer fabrication techniques [Chow et al., 2007; Hyysalo et al., 2017; Zuidema et al., 2018] have all been shown to orient astrocyte 
Table 4. In vitro response of astrocytes to surface topography

\begin{tabular}{|c|c|c|c|}
\hline Surface topography & Type of astrocytes & Astrocytic changes & Reference \\
\hline Nanoporous gold & Rat cortical & Reduction of astrocyte coverage & $\begin{array}{l}\text { Chapman et al., } 2015 \\
\text { Chapman et al., } 2016\end{array}$ \\
\hline Nanoporous alumina & Rat cortical & $\begin{array}{l}\text { Pore size altered astrocyte attachment, focal } \\
\text { adhesions, and GFAP expression }\end{array}$ & Ganguly et al., 2018 \\
\hline Porous PCL and PDMS & Rat cortical & Bridging of pores & Sun et al., 2011 \\
\hline Porous Alvatex & Mouse cortical & Retention of nonreactive phenotype & Ugbode et al., 2016 \\
\hline $\begin{array}{l}\text { Rough silica nanoparticle } \\
\text { surface }\end{array}$ & Rat hippocampal & Independent growth of astrocytes and neurons & Blumenthal et al., 2014 \\
\hline Nanostructured $\mathrm{TiO}_{2}$ & Rat cortical & Increased production of IL-10, MMP-8, BDNF & De Astis et al., 2013 \\
\hline Carbon nanofiber & DI TNC1 & Reduced proliferation & McKenzie et al., 2004 \\
\hline PEG-SWCNTs & Mouse cortical & $\begin{array}{l}\text { Round growth, } \downarrow \text { GFAP expression, } \uparrow \text { proliferation, } \\
\text { GFAP dependent }\end{array}$ & $\begin{array}{l}\text { Gottipati et al., } 2013 \\
\text { Gottipati et al., } 2014\end{array}$ \\
\hline Silicon pillar array & Rat cortical & Astrocytes grew above pillars & Turner et al., 2000 \\
\hline Soft silicone pillars & Rat cortical & $\begin{array}{l}\text { 4.7- } \mu \mathrm{m} \text { pillars engulfed and bent, inhibition of } \\
\text { migration and proliferation on tall pillars }\end{array}$ & Minev et al., 2013 \\
\hline Silica/parylene-C grids & Human NT2 derived & Growth in grid patterns & Jordan et al., 2016 \\
\hline PMMA microgrooves & C6 rat astrocytoma & Inhibition of GFAP expression & Ereifej et al., 2013 \\
\hline PDMS 1,000-nm grooves & Rat cortical & $\begin{array}{l}\text { Enhanced mitochondrial activity, ATP release, } \\
\text { calcium peaks and release }\end{array}$ & Singh et al., 2015 \\
\hline Micropatterned PMMA & Mouse cortical & $\begin{array}{l}\text { Expression of markers and elongation similar to } \\
\text { radial glia }\end{array}$ & Mattotti et al., 2012 \\
\hline
\end{tabular}

growth parallel to the alignment presented by micro- and nanotopographies. These studies demonstrate the ability of astrocytes to sense the presence of topographical structures, leading to experiments that have begun to elucidate the astrocyte biological response to surface topography.

Porous surfaces are being developed for applications in the spinal cord due to their ability to allow for enhanced transport of nutrients [Yang et al., 2005], to alter cellular adhesion and growth [Namba et al., 2009], and/ or improve electrode properties [Silva et al., 2014]. Gold electrodes are under development for use in the nervous system due to their biofouling-resistant electrical performance, high fidelity recordings, high effective surface area, and tunable pore sizes [Chapman et al., 2015]. Nanoporous gold surfaces seeded with both astrocytes and neurons show selective reduction of astrocytic coverage while maintaining high neuronal coverage [Chapman et al., 2015]. This response was later revealed to be due to both an initial inhibition of astrocyte attachment and topographical cues provided by the nanoporous gold struc- ture [Chapman et al., 2016]. Nanoporous alumina surfaces have also been studied, and it was found that pore size was important for astrocyte attachment, focal adhesions, and GFAP expression [Ganguly et al., 2018]. Astrocyte growth into pores on the scale of hundreds of microns was studied in vitro to understand how these cells may interact with implanted porous conduits. Astrocytes were found to bridge the pores once they were fully confluent around the porous structures [Sun et al., 2011]. Porous Alvatex ${ }^{\circledR} 3 \mathrm{D}$ scaffolds were used to study how both embryonic and postnatal murine cortical astrocytes respond to porous substrates. Embryonic astrocytes showed decreases in protein expression of GFAP, NG2, GLAST, and GLT-1 compared to 2D cultures, while only SMC3 was downregulated in postnatal astrocytes [Ugbode et al., 2016]. These results suggest that the selection of the proper astrocyte source is important for any study addressing astrocyte response to surfaces. In order to gain a greater understanding of the astrocyte response to porous structures, studies that detail astrocyte gene and protein ex- 
pression changes in response to pore size, porosity, and pore thickness should be undertaken.

Surface roughness is another important parameter to consider when engineering biomaterials for applications in the spinal cord. Surfaces with a nanoroughness range from root mean square roughness $\left(\mathrm{R}_{\mathrm{q}}\right)$ of $3.5-80 \mathrm{~nm}$ fabricated by spin-coating of silica nanoparticles, and surfaces with an $\mathrm{R}_{\mathrm{q}}$ of $32 \mathrm{~nm}$, showed rat hippocampal neurons and astrocytes growing independently of each other [Blumenthal et al., 2014]. These findings suggest a link between surface roughness and astrocyte response that alters neuronal growth. Nanostructured $\mathrm{TiO}_{2}$ surfaces with $R_{q}$ of both 20 and $29 \mathrm{~nm}$ were generated to study the astrocyte production of inflammatory factors [De Astis et al., 2013]. It was observed that surface roughness does not enhance the cortical astrocyte production of proinflammatory factors, but does increase their production of interleukin-10, matrix metalloprotease 8 , and brain-derived neurotrophic factor. Neurons did associate with astrocytes in this study, even though the surface roughness was similar to the 32-nm silica surface, showing the complex array of factors involved in astrocyte response to surfaces. Carbon nanofibers possess excellent conductivity properties [Lozano, 2000], making them a potential electrode coating material. Studying the astrocyte response to carbon nanofibers of $60-100 \mathrm{~nm}$ or $125-200 \mathrm{~nm}$ in vitro, it was revealed that astrocyte proliferation and function was minimized on these surfaces [McKenzie et al., 2004].

Single-walled carbon nanotube (SWCNT) films have well understood electrical properties that can influence the electrical properties of neurons [Mazzatenta et al., 2007]. SWCNTs can be readily functionalized with PEG, improving their biocompatibility. Therefore, PEG-functionalized SWCNT films of different thicknesses $(10,30$, and $60 \mathrm{~nm}$ ) were used to modulate the morphology, function, and proliferative characteristics of astrocytes [Gottipati et al., 2013]. Astrocytes were found to grow bigger and rounder in shape on various thicknesses of SWCNTs, showed lower expression of GFAP, and had an increase in proliferation. These results suggest that PEG-SWCNTs may reduce astrogliosis in vivo. Using astrocytes from GFAP null mice, the changes associated with PEGSWCNTs were shown to be dependent on astrocyte GFAP expression [Gottipati et al., 2014]. These studies demonstrate that surface roughness is another important factor to consider when developing biomaterials that interact with astrocytes.

Nano- and micropatterned surfaces have been developed to study the astrocyte response to different topographical structures. One of the earliest studies showed that astrocytes cultured on silicon pillar arrays made contact with the pillars but did not reach down into 1 - to 5 - $\mu \mathrm{m}$ inter-pillar gaps [Turner et al., 2000]. This led to the study of the astrocyte response to soft silicone micropillars. Astrocytes were shown to engulf and bend micropillars with a height of $4.7 \mu \mathrm{m}$, but not shorter pillars of 0.5 $\mu \mathrm{m}$ [Minev et al., 2013]. Also, astrocytes cultured on the tall pillars were less migratory and proliferative on these pillars, suggesting that surface patterning can be used to alter astrocyte behavior. Grid networks composed of $\mathrm{SiO}_{2}$ grids inlaid with biocompatible parylene- $\mathrm{C}$ have also been developed to study astrocyte interactions from the single cell to the network level [Jordan et al., 2016]. While more work needs to be done in this field, the study showed the feasibility of this approach to pattern astrocytes in a grid network. Grooved channels have also been used to study how these structures impact astrocytes. Grooves $277 \mathrm{~nm}$ wide with a period of 3,600 grooves $/ \mathrm{mm}$ patterned in poly(methyl methacrylate) PMMA were developed to study astrocyte reactivity. These grooves were found to promote astrocyte alignment, reduce cell adhesion and proliferation, and to inhibit GFAP protein expression [Ereifej et al., 2013]. Polydimethylsiloxane grooves with $1,000-\mathrm{nm}$ spacing and 250 - to $500-\mathrm{nm}$ depths were used to study astrocyte calcium signaling. Again, these grooves aligned astrocyte growth, cytoskeletal components, and the nucleus-centrosome axis [Singh et al., 2015]. Astrocytes also showed enhanced mitochondrial activity, ATP release, and calcium peaks and release, suggesting that the response of astrocytes to surface patterning is a complex process that may impact the entire cell. Mattotti et al. [2012] used micropatterned PMMA substrates to study the astrocyte response to lanes of both 2 and $10 \mu \mathrm{m}$. Using P0 mouse cortical astrocytes, this study found evidence to suggest that astrocytes grown on 2- $\mu \mathrm{m}$ PMMA substrates express markers and elongate similarly to a radial glial cell phenotype. These cells were in close association with cultured neurons and directed neurite outgrowth. While these studies represent an important step forward in understanding the response of astrocytes to patterned surfaces, much more work is needed to elucidate changes in gene and protein expression in order to begin to develop a broader understanding of how astrocytes respond to different surface patterns.

Electrospun polymer fibers have been developed extensively for tissue engineering applications because of their capacity to mimic the topography of the ECM [Ingavle and Leach, 2014]. The ability to use multiple biodegradable polymers, readily control fiber diameter, and ease of aligning the fibers make them especially attractive 
Table 5. In vitro response of astrocytes to electrospun fibers

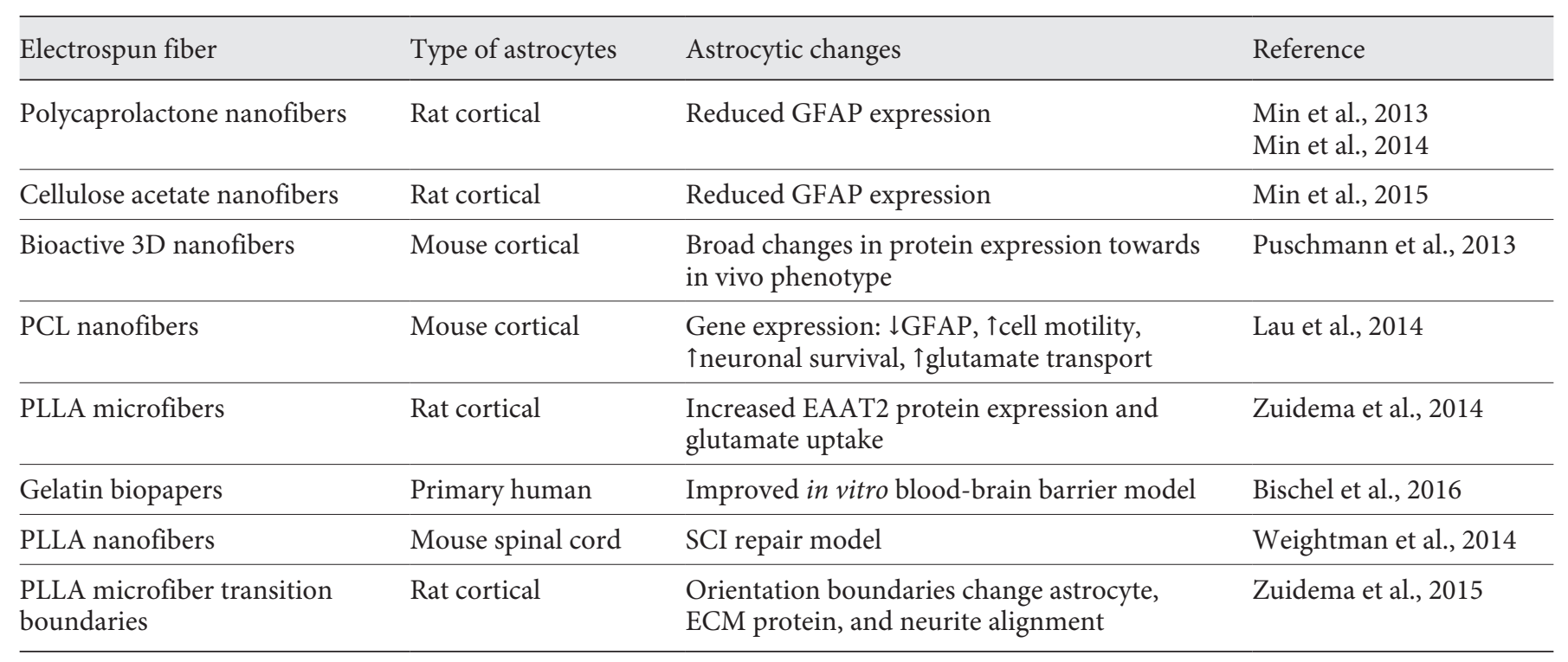

for nervous system applications [Schaub et al., 2016]. Because of this, several studies have examined the ability of fibers to alter astrocyte behavior in culture (Table 5) [Baiguera et al., 2010; Schaub and Gilbert, 2011; Kim et al., 2012; Qu et al., 2013]. One important parameter studied is the expression of GFAP, where astrocytes seeded on both polycaprolactone (PCL) nanofibers [Min et al., 2013, 2014] and cellulose acetate nanofibers [Min et al., 2015] decreased their expression of GFAP compared to tissue culture plastic controls. In order to gain a better understanding of how astrocytes are reacting to polymer fibers, more in-depth biological analysis was conducted. Using a polyether-based polyurethane resin, fibers with an average diameter of $1,200 \mathrm{~nm}$ were created. Puschmann et al. [2013] found that these fibers again reduced GFAP protein expression of primary cortical astrocytes following 6 days in culture. Importantly, the 1,200-nm fibers used had extensive effects on the cultured astrocytes. Astrocytes cultured on the 1,200-nm fibers showed lower protein expression of nestin, synemin, vimentin, and heat shock protein 70, suggesting that the 3D nanofiber topography creates a less stressful environment for the astrocytes. They then studied the gene expression profiles of these astrocytes and broadly found that gene expression differed in biochemical pathways that regulate cell proliferation, cell shape, and cell motility. Lau et al. [2014] studied the effects of both random and aligned PCL nanofibers on mouse astrocyte gene and protein expression at both 4 and 12 days in vitro. Again, GFAP protein expression was downregulated when astrocytes were grown on the PCL nanofiber topography. mRNA expression of genes involved in cell motility and pathfinding (skeletal muscle actin- $\alpha 2$, vinculin, and chemokine [C-X-C motif] ligand 12), promotion of neuronal survival (brain-derived neurotrophic factor, anti-oxidant [glutathione Stransferase $\alpha 1$ ], and heme oxygenase 1), and glutamate transporter (EAAT2) were all upregulated when astrocytes were cultured on PCL nanofibers. Furthermore, primary rat astrocytes cultured on poly-L-lactic acid electrospun fibers (with diameters equal to $2 \mu \mathrm{m}$ ) showed increased immunohistochemical and protein expression of EAAT2, while functionally taking up more glutamate in solution compared with astrocytes cultured on $2 \mathrm{D}$ surfaces [Zuidema et al., 2014]. These two studies suggest that astrocytes are not only less reactive when in contact with polymer fibers, but that astrocytes in contact with microfibers may be growth supportive for neurons.

Electrospun fibers have also been used to create in vitro models of the blood-brain barrier, and the astrocyte environment following SCI. Electrospun gelatin biopapers were used to create an in vitro blood-brain barrier model [Bischel et al., 2016]. Cocultures of human endothelial cells and astrocytes on gelatin biopapers had improved transendothelial electrical resistance, decreased permeability, and permitted a smaller separation between cells compared to the standard polyethylene terephthalate inserts. Electrospun fibers have also been used to study in vitro SCI models. 3D organotypic spinal cord slice arrays were connected to poly-lactic acid nanofiber meshes to study cellular growth induced by the nanofi- 
bers [Weightman et al., 2014]. The aligned fibers directed astrocyte growth, and neurons were found associated with the oriented astrocytes. Another in vitro model produced a transition boundary of aligned astrocytes with nonoriented astrocytes by removing aligned poly-L-lactic acid fibers using chloroform [Zuidema et al., 2015]. This created a gap composed of a polymer film bordering aligned fibers. Astrocytes were seeded on these surfaces, followed by dorsal root ganglia neurons. It was found that the aligned astrocytes directed neurite extension, but if a neurite crossed into the nonoriented area, neurites lost their aligned growth and began turning. While these studies may not directly study how astrocytes are being impacted by biomaterials, they do show that there are many potential in vitro applications for polymer fibers.

\section{Analysis of Astrocyte Response to Biomaterials Implanted into Preclinical Animal Models of SCI}

Extensive astrocyte in vitro culture experiments have been conducted using various biomaterials to study the ability of the biomaterial to change astrocyte behavior. Although these studies have provided some insight, these findings should be extended to an appropriate in vivo SCI model to test if the biomaterial induces astrocyte differentiation towards specific reactive states. Here, we review manuscripts that have used biomaterial approaches to assess glial reactivity, axonal regeneration, and functional recovery. Since our focus is particularly on astrocytes, we only include the biomaterial approaches that have studied the effects of the material on astrocytes and have shown a change in astrocytic properties (Table 6).

\section{Natural Biomaterials and Synthetic Biomaterials}

Hydrogels and other injectable biomaterials hold great promise in the field of regenerative medicine [Kretlow et al., 2007; Hoare and Kohane, 2008; Klouda and Mikos, 2008]. Trauma to the spinal cord typically leads to the formation of an irregular cavity. Injectable biomaterials, hydrogels in particular, can fill these cavities and act as a scaffold for cellular infiltration and matrix deposition. The mechanical properties of the hydrogel scaffolds can be adjusted to match the properties of the native spinal cord tissue, compared to most preformed biomaterial matrices. In addition, injectable materials can be mixed, in their liquid state, with therapeutics or growth factors prior to their injection into the spinal cord, and hence can act as drug delivery agents. Currently, continuous or prolonged infusion of medication for pain relief is facilitated through the use of catheters and mini pumps directly into the spinal cord or the surrounding tissue [Tutak and Doleys, 1996]. However, these infusion strategies are prone to blockages and/or infections [Amar et al., 2005]. Hence, injectable hydrogel materials can controllably release medication over time. The release characteristics of the loaded therapeutics can be modulated by adjusting the physical and chemical properties of the injectables or by incorporating secondary vehicles such as micro- and nanoparticles, liposomes, and microtubules [Kretlow et al., 2007; Willerth and Sakiyama-Elbert, 2007]. We review some of the studies that have focused on injectable hydrogel biomaterials for SCI applications either as a scaffold, a drug delivery agent, or both.

Hydrogels, being injectable, make an excellent biomaterial for translational applications, particularly in SCI applications where the damage to the spinal cord typically leaves a cavity devoid of any tissue [Woerly, 2000]. Several studies have used hydrogels to study their effects on tissue regeneration following an SCI. In the following sections, we highlight hydrogel strategies used within in vivo animal models.

\section{Natural Hydrogels}

Joosten et al. [1995] have shown that in situ gelling collagen scaffolds reduce the accumulation of astrocytes at the interface but still promote ingrowth of the accumulated astrocytes into the scaffold 4 weeks after implantation in a T8-T9 dorsal hemisection rat SCI model. This, however, was not observed when a preassembled collagen gel was used instead, and the authors report that an optimal integration between host and graft is important to promote the ingrowth of astrocytes into the biomaterial. The work by Marchand and Woerly [1990] has also shown that in situ gelling collagen hydrogel promotes astrocytic ingrowth followed by axons into the biomatrix, 3 months after implantation in a T8-T10 complete transection rat SCI. However, the collagen matrix denatured in 2-3 months. So, to improve the stability and durability of the collagen, in a subsequent study, Marchand et al. [1993] coprecipitated collagen with chondroitin-4-sulfate or chemically crosslinked the collagen with carbodiimide and implanted them into a T8-T10 hemisection rat SCI model. They showed that the hydrogels have improved mechanical properties and favor axonal regeneration by modifying the normal scarring process as assessed by type 1 collagen deposition in the scar 4 weeks and 6 months postimplantation. The use of a collagen impregnated acrylic hydrogel made of 2-hydroxyethyl methacrylate implanted into $\mathrm{T} 8$ - $\mathrm{T} 9$ suction cavities of the dorsal 
Table 6. In vivo response of astrocytes to various biomaterials

\begin{tabular}{|c|c|c|c|}
\hline Biomaterial used & Type of injury & Astrocytic changes & Reference \\
\hline Collagen hydrogel & T8-T9 rat dorsal hemisection & Ingrowth of astrocytes & Joosten et al., 1995 \\
\hline Collagen acrylic hydrogel & $\begin{array}{l}\text { T8-T9 rat suction cavities of } \\
\text { the dorsal funiculus }\end{array}$ & $\begin{array}{l}\text { Minimal astrocytic } \\
\text { accumulation at the interface }\end{array}$ & Giannetti et al., 2001 \\
\hline Fibrin hydrogel & T8 rat complete transection & Reduced GFAP-ir & Taylor et al., 2006 \\
\hline $\begin{array}{l}\text { Self-assembling peptide } \\
\text { amphiphiles }\end{array}$ & T10 mice clip compression & Reduced GFAP-ir & $\begin{array}{l}\text { Tysseling-Mattiace et al., } \\
2008\end{array}$ \\
\hline Neurogel & $\begin{array}{l}\text { T6-T7 cat complete } \\
\text { transection }\end{array}$ & Prevention of scar formation & Woerly et al., 2001a, 2004 \\
\hline Neurogel & $\begin{array}{l}\text { T6-T7 cat complete } \\
\text { transection }\end{array}$ & Ingrowth of astrocytes & Woerly et al., 2001b \\
\hline $\begin{array}{l}\text { Collagen hydrogel with EGF } \\
\text { and basic FGF-2 }\end{array}$ & $\mathrm{T} 2$ rat severe compression & $\begin{array}{l}\text { Increased GFAP-ir and } \\
\text { decreased lesion cavity }\end{array}$ & Jimenez Hamann et al., 2005 \\
\hline Agarose hydrogel with BDNF & T10 rat dorsal hemisection & $\begin{array}{l}\text { Reduced GFAP-ir and CSPG } \\
\text { production }\end{array}$ & Jain et al., 2006 \\
\hline $\begin{array}{l}\text { Agarose hydrogel with } \\
\text { MP-loaded nanoparticles }\end{array}$ & T9-T10 rat contusion & Decreased lesion volume & Chvatal et al., 2008 \\
\hline $\begin{array}{l}\text { Agarose hydrogel with } \\
\text { chABC-loaded lipid microtubes }\end{array}$ & T10 rat dorsal hemisection & $\begin{array}{l}\text { Decreased number of reactive } \\
\text { astrocytes }\end{array}$ & Lee et al., 2010 \\
\hline $\begin{array}{l}\text { Aligned poly-L-lactic acid } \\
\text { microfibers }\end{array}$ & $\begin{array}{l}\text { T9-T10 rat complete } \\
\text { transection }\end{array}$ & Ingrowth of astrocytes & Hurtado et al., 2011 \\
\hline Collagen nanofibers & C3 rat hemisection & No astrocyte infiltration & Liu et al., 2012 \\
\hline $\begin{array}{l}\text { Poly(lactic-co-glycolic acid) and } \\
\text { poly( } \varepsilon \text {-caprolactone }) \text { channels }\end{array}$ & T10 rat contusion & Minimal astrocyte infiltration & Gelain et al., 2011 \\
\hline $\begin{array}{l}\text { Poly(propylene carbonate) } \\
\text { microfibers with dbcAMP }\end{array}$ & T8 rat hemisection & Decreased glial scar thickness & Xia et al., 2013 \\
\hline
\end{tabular}

funiculus of adult rat spinal cords showed minimal astrocytic accumulation at the interface, which was not arranged in dense rows, and no cystic cavitation accompanied by axonal penetration along the full length of the hydrogel [Giannetti et al., 2001].
High molecular weight HA-based hydrogels designed to resist degradation were implanted into a rat T7-T8 dorsal hemisection injury model and was shown to effectively reduce astrocyte proliferation, GFAP immunoreactivity (ir), and CSPG production in vivo [Khaing et al., 
2011]. This study demonstrated that HA-based hydrogels, when stabilized against degradation, can be used to minimize undesired scarring unlike the native HA, which was shown to degrade and induce the activation and proliferation of astrocytes [Struve et al., 2005].

Fibrin scaffolds significantly enhance the regeneration environment by decreasing the astroglial scar formation at the white matter border of the lesion in a T8 complete transection rat SCI based on GFAP-ir at 9 days postimplantation [Taylor et al., 2006]. Delayed implantation of prepolymerizing fibrin scaffolds, however, implanted 2 weeks after a T9 dorsal hemisection in rats delayed the accumulation of GFAP-positive astrocytes at the lesion border and lessened the migration of astrocytes into the lesion, although no change in CSPG deposition was reported [Johnson et al., 2010].

\section{Synthetic Injectable Biomaterials}

Injectable, self-assembling peptide amphiphiles (PA) incorporating the neuroactive pentapeptide epitope from laminin, isoleucine-lysine-valine-alanine-valine (IKVAV), were used by Tysseling-Mattiace et al. [2008] because of their ease of self-assembly in vivo and the ability of IKVAV PA to promote neurite outgrowth in culture [Silva et al., 2004]. They showed that the injection of IKVAV PA into mice following an acute T10 clip compression SCI caused a significant reduction in GFAP-ir and facilitated the regeneration of ascending and descending sensory axons at the lesion site at 11 weeks postinjection. While regeneration occurred, there was no change in functional recovery compared to the controls.

Chemically functionalized SWCNTs promoted neurite extension of hippocampal neurons in culture [ $\mathrm{Ni}$ et al., 2005]. This finding was extended to an in vivo study where SWCNTs functionalized with PEG was injected into the lesion following a T9 rat complete transection SCI [Roman et al., 2011]. The treatment significantly decreased the lesion volume without increasing reactive astrogliosis. A significant improvement in hindlimb locomotor recovery was also reported associated with an increase in the number of corticospinal tract fibers.

\section{Synthetic Hydrogels}

Neurogel, a biocompatible poly (N-[2-hydroxypropyl] methacrylamide) hydrogel, promoted axonal growth into the matrix in part by reducing the reactive astroglial response and preventing scar formation as assessed using GFAP-ir 6 months postimplantation in a cat T6-T7 complete transection SCI [Woerly et al., 2001a, 2004]. This was a follow-up study to work done in rats using delated implantation of Neurogel (14 weeks postinjury) where similar ingrowth of astrocytes and axons was observed 7 months postimplantation along with significant improvement in functional recovery [Woerly et al., 2001b].

Drug Delivery from Injectable Hydrogel Systems

In addition to providing a matrix for tissue regeneration, hydrogels can also be developed to release drugs locally to the injury site. A combination of epidermal growth factor and basic FGF-2 loaded into a collagen hydrogel was used by Jimenez Hamann et al. [2005] to stimulate the proliferation and differentiations of ependymal cells, a potential source of endogenous progenitor/stem cells in the adult spinal cord. They showed that the hydrogel did increase ependymal cell proliferation following intrathecal injection in a severe compressive rat SCI model at the T2 level. However, the authors reported an increase in the GFAP-ir with a decrease in the lesion cavity. The growth factor BDNF (brain-derived neurotrophic factor) loaded into an agarose-based hydrogel reduced the reactivity of astrocytes, as assessed by a reduction in GFAP-ir, and the production of CSPGs following implantation in a T10 dorsal hemisection rat model [Jain et al., 2006]. A significant increase in axonal regeneration and sprouting into the hydrogel was also reported.

The glucocorticoid methylprednisolone (MP) is the only FDA-approved drug for SCI applications [Bracken et al., 1998]. MP was shown to improve neurological recovery in humans if administered within $8 \mathrm{~h}$ after SCI [Bracken et al., 1990]. However, a high system dose of MP causes adverse side effects, including wound infections, pneumonia, and acute corticosteroid myopathy, and these side effects would be minimized by controlled and localized delivery of MP [Bracken et al., 1990; Gerndt et al., 1997; Legos et al., 2001; Qian et al., 2005]. Work by Chvatal et al. [2008] encapsulated MP in biodegradable poly(lactic-co-glycolic acid)-based nanoparticles in agarose hydrogels. This injectable composite was placed into a T9-T10 contusion rat SCI. Following delivery of the MP-containing hydrogel, the authors noted a significant reduction in lesion volume 7 days postinjury. A follow-up study by the same group using MP-loaded nanoparticles in an agarose matrix enabled a significant reduction in GFAP-ir and CSPG-ir at the lesion border in the presence of the hydrogel, 2 and 4 weeks postimplantation in a T9T10 rat dorsal hemisection SCI model [Kim et al., 2009].

Another anti-inflammatory agent, minocycline, was loaded into an injectable Tetronic-oligolactide copolymer hydrogel, which is liquid at room temperature and 
solidifies at $37^{\circ} \mathrm{C}$. Injection of this hydrogel in the lesion created following a rat T7 hemisection SCI significantly decreased CSPG deposition and increased axonal ingrowth at 1 and 6 weeks postinjection, respectively [Kang et al., 2010].

Ren et al. [2014] reported a decrease in GFAP-ir and an increase in neuronal survival and regeneration using the broad-spectrum cell cycle inhibitor Flavopiridol loaded into poly(lactic-co-glycolic acid) nanoparticles injected into a lesion created by a hemisection SCI at the T10 level in rats. In addition, a reduction in the cavitation by approximately $90 \%$ was also reported along with improved motor recovery.

Since the accumulation of axon-inhibiting CSPGs occurs in the glial scar, chondroitinase $\mathrm{ABC}$ (chABC) was used in several studies to digest the GAG chains on CSPGs and thereby overcome CSPG-mediated inhibition [Bradbury et al., 2002; Pizzorusso et al., 2002, 2006; Barritt et al., 2006]. Since chABC loses its activity rapidly at physiological temperature [Tester et al., 2007], a thermostabilized chABC was made by Lee et al. [2010] using the sugar trehalose. The chABC was loaded into lipid microtubes embedded in an agarose hydrogel and injected in a rat T10 dorsal hemisection SCI. The thermostabilized chABC was reported to be stable for at least 2 weeks and has shown successful CSPG digestion following implantation in vivo. The hydrogel also decreased the number of reactive astrocytes at the lesion site 2 weeks after injury as assessed using GFAP-ir.

\section{Topographical Guidance Scaffolds}

Although several groups have focused on using injectable materials as SCI therapies, many are studying fibrous materials within animal models of SCI. Fibrous materials provide a physical scaffold for the growing cells. These scaffolds are designed to mimic the fascicular nerve architecture, and the fibrous ECM within native tissue in terms of both chemical composition and physical structure in addition to being biocompatible, nontoxic, nonmutagenic, and nonimmunogenic [Goyal et al., 2016]. Furthermore, they provide appropriate mechanical support and show favorable topographical properties to improve cell adhesion, proliferation, and differentiation [Lanza et al., 2011]. In addition to acting as a scaffold, they can also be used for drug delivery applications similar to injectable biomaterials.

Electrospun Fiber Guidance Scaffolds

Electrospun fibers made from different biocompatible polymers were used to direct the migration of astrocytes and the extension of neurites from neurons in culture. Some of these materials were tested in vivo to assess their effects on the tissue following a SCI. Hurtado et al. [2011] used conduits made of highly aligned electrospun poly-Llactic acid microfibers (fiber diameter of 1.2-1.6 $\mu \mathrm{m}$ ) and implanted them in a T9-T10 complete transection rat SCI model. Astrocytes were shown to migrate into the conduits along the length of the fibers 4 weeks postimplantation and axonal regeneration was localized to these migration astrocytes. Work using electrospun collagen nanofibers in a C3 hemisection rat SCI has shown significant neural fiber sprouting 30 days postimplantation [Liu et al., 2012]. However, no infiltration of astrocytes into the conduits was reported. Self-assembling peptides assembled into electrospun guidance channels made of poly(lactic-co-glycolic acid) and poly( $\varepsilon$-caprolactone) have been used to study functional recovery in a delayed implantation T10 contusion SCI in rats [Gelain et al., 2011]. The channels were implanted into the cysts formed after a contusion SCI at 1 month postinjury. Astrocytes were shown to surround the implant with very minimal infiltration into the tubular channels. There was also a significant ingrowth of $\beta$ III-tubulin-positive neurons in the scaffolds. A study by Xia et al. [2013] reported on the sustained delivery of dibutyryl cyclic adenosine monophosphate to the hemisected spinal cord in rats at the T8 level from electrospun poly(propylene carbonate) microfibers. Four weeks postimplantation, the scaffold decreased the thickness of the glial scar, assessed using GFAP labeling, and promoted axonal growth into the scar.

\section{Conclusion}

This review highlights the advances made in the field of SCI therapies using biomaterials. Although significant progress is being made using different materials, there are only limited studies that show an improvement in functional recovery. One important and relatively unexplored area for the advancement of biomaterial approaches could be to specifically target astrocytes, which in turn can drive axonal regeneration. A time-dependent analysis of the glial scarring response has shown positive and protective effects of astrocytes at the acute stage of injury. Subsequently, the astrocytes shift to a negative and inhibitory barrier suppressing axonal regeneration at the chronic stage of injury [Rolls et al., 2009]. Hence, biomaterial approaches can be designed to revert astrocytes back to a neuron-supportive phenotype. This idea is sup- 
ported by recent advances in the field that show that environmental cues can drive the astrocyte phenotype in vivo after injury and the process of scar formation could be reversible [Hara et al., 2017]. A different approach could be to better understand the differences between reactive states of astrocytes and use biomaterials to target the mechanisms underlying the induction of those reactive states [Liddelow and Barres, 2017]. A drive from a detrimental phenotype to a neuroprotective phenotype may boost axonal regeneration.

\section{Acknowledgements}

The authors thank Blair Cooper for his help in creating the figures.

\section{Statement of Ethics}

The authors have no ethical conflicts to disclose.

\section{Disclosure Statement}

The authors have no conflicts of interest to declare.

\section{Funding Sources}

The authors acknowledge the following funding support: an NIH R01 Grant (NS092754) and New York State Spinal Cord Injury Research Board (NYSSCIRB) Institutional Support Grant (C32245GG) to R.J.G., and Craig H. Neilsen Foundation (468116) to M.K.G.

\section{References}

Agulhon, C., J. Petravicz, A.B. McMullen, E.J. Sweger, S.K. Minton, S.R. Taves, K.B. Casper, T.A. Fiacco, K.D. McCarthy (2008) What is the role of astrocyte calcium in neurophysiology? Neuron 59: 932-946.

Almad, A., F.R. Sahinkaya, D.M. McTigue (2011) Oligodendrocyte fate after spinal cord injury. Neurotherapeutics 8: 262-273.

Amar, A.P., D.W. Larsen, G.P. Teitelbaum (2005) Percutaneous spinal interventions. Neurosurg Clin N Am 16: 561-568.

Anderson, C.M., R.A. Swanson (2000) Astrocyte glutamate transport: review of properties, regulation, and physiological functions. Glia 32: 1-14.

Anderson, M.A., Y. Ao, M.V. Sofroniew (2014) Heterogeneity of reactive astrocytes. Neurosci Lett 565: 23-29.

Anderson, M.A., J.E. Burda, Y. Ren, Y. Ao, T.M. O'Shea, R. Kawaguchi, G. Coppola, B.S. Khakh, T.J. Deming, M.V. Sofroniew (2016) Astrocyte scar formation AIDS central nervous system axon regeneration. Nature 532: 195-200.

Annabi, N., A. Tamayol, J.A. Uquillas, M. Akbari, L.E. Bertassoni, C. Cha, G. Camci-Unal, M.R. Dokmeci, N.A. Peppas, A. Khademhosseini (2014) 25th anniversary article: rational design and applications of hydrogels in regenerative medicine. Adv Mater 26: 85-124.

Azemi, E., W.R. Stauffer, M.S. Gostock, C.F. Lagenaur, X.T. Cui (2008) Surface immobilization of neural adhesion molecule L1 for improving the biocompatibility of chronic neural probes: in vitro characterization. Acta Biomater 4: 1208-1217.

Bahney, J., C.S. von Bartheld (2018) The cellular composition and glia-neuron ratio in the spinal cord of a human and a nonhuman primate: comparison with other species and brain regions. Anat Rec 301: 697-710.
Baiguera, S., C. Del Gaudio, L. Fioravanzo, A. Bianco, M. Grigioni, M. Folin (2010) In vitro astrocyte and cerebral endothelial cell response to electrospun poly( $\varepsilon$-caprolactone) mats of different architecture. J Mater Sci Mater Med 21: 1353-1362.

Balasubramanian, S., J.A. Packard, J.B. Leach, E.M. Powell (2016) Three-dimensional environment sustains morphological heterogeneity and promotes phenotypic progression during astrocyte development. Tissue Eng Part A 22: 885-898.

Bareyre, F.M., M.E. Schwab (2003) Inflammation, degeneration and regeneration in the injured spinal cord: insights from DNA microarrays. Trends Neurosci 26: 555-563.

Barrett, C.P., L. Guth, E.J. Donati, J.G. Krikorian (1981) Astroglial reaction in the gray matter of lumbar segments after midthoracic transection of the adult rat spinal cord. Exp Neurol 73: 365-377.

Barritt, A.W., M. Davies, F. Marchand, R. Hartley, J. Grist, P. Yip, S.B. McMahon, E.J. Bradbury (2006) Chondroitinase ABC promotes sprouting of intact and injured spinal systems after spinal cord injury. J Neurosci 26: 1085610867.

Beattie, M.S., A.A. Farooqui, J.C. Bresnahan (2000) Review of current evidence for apoptosis after spinal cord injury. J Neurotrauma 17: 915-925.

Bi, F., C. Huang, J. Tong, G. Qiu, B. Huang, Q. Wu, F. Li, Z. Xu, R. Bowser, X.G. Xia, H. Zhou (2013) Reactive astrocytes secrete lcn2 to promote neuron death. Proc Natl Acad Sci USA 110: 4069-4074.

Bignami, A., D. Dahl (1974) Astrocyte-specific protein and neuroglial differentiation: an immunofluorescence study with antibodies to the glial fibrillary acidic protein. J Comp Neurol 153: 27-37.
Biran, R., M.D. Noble, P.A. Tresco (1999) Characterization of cortical astrocytes on materials of differing surface chemistry. J Biomed Mater Res 46: 150-159.

Bischel, L.L., P.N. Coneski, J.G. Lundin, P.K. Wu, C.B. Giller, J. Wynne, B.R. Ringeisen, R.K. Pirlo (2016) Electrospun gelatin biopapers as substrate for in vitro bilayer models of bloodbrain barrier tissue. J Biomed Mater Res A 104: 901-909.

Blumenthal, N.R., O. Hermanson, B. Heimrich, V.P. Shastri (2014) Stochastic nanoroughness modulates neuron-astrocyte interactions and function via mechanosensing cation channels. Proc Natl Acad Sci USA 111: $16124-$ 16129.

Bracken, M.B., M.J. Shepard, W.F. Collins, T.R. Holford, W. Young, D.S. Baskin, H.M. Eisenberg, E. Flamm, L. Leo-Summers, J. Maroon, L.F. Marshall, P.L. Perot, J. Piepmeier, V.K.H. Sonntag, F.C. Wagner, J.E. Wilberger, H.R. Winn (1990) A randomized, controlled trial of methylprednisolone or naloxone in the treatment of acute spinal-cord injury. N Engl J Med 322: 1405-1411.

Bracken, M.B., M.J. Shepard, T.R. Holford, L. Leo-Summers, E.F. Aldrich, M. Fazl, M.G. Fehlings, D.L. Herr, P.W. Hitchon, L.F. Marshall, R.P. Nockels, V. Pascale, P.L. Perot, J. Piepmeier, V.K. Sonntag, F. Wagner, J.E. Wilberger, H.R. Winn, W. Young (1998) Methylprednisolone or tirilazad mesylate administration after acute spinal cord injury: 1-year follow up: results of the third National Acute Spinal Cord Injury randomized controlled trial. J Neurosurg 89: 699-706.

Bradbury, E.J., L.D.F. Moon, R.J. Popat, V.R. King, G.S. Bennett, P.N. Patel, J.W. Fawcett, S.B. McMahon (2002) Chondroitinase ABC promotes functional recovery after spinal cord injury. Nature 416: 636-640. 
Burda, J.E., A.M. Bernstein, M.V. Sofroniew (2016) Astrocyte roles in traumatic brain injury. Exp Neurol 275: 305-315.

Bush, T.G., N. Puvanachandra, C.H. Horner, A. Polito, T. Ostenfeld, C.N. Svendsen, L. Mucke, M.H. Johnson, M.V. Sofroniew (1999) Leukocyte infiltration, neuronal degeneration, and neurite outgrowth after ablation of scarforming, reactive astrocytes in adult transgenic mice. Neuron 23: 297-308.

Chapman, C.A.R., H. Chen, M. Stamou, J. Biener, M.M. Biener, P.J. Lein, E. Seker (2015) Nanoporous gold as a neural interface coating: Effects of topography, surface chemistry, and feature size. ACS Appl Mater Interfaces 7: 7093-7100.

Chapman, C.A.R., H. Chen, M. Stamou, P.J. Lein, E. Seker (2016) Mechanisms of reduced astrocyte surface coverage in cortical neuron-glia co-cultures on nanoporous gold surfaces. Cell Mol Bioeng 9: 433-442.

Chaubaroux, C., F. Perrin-Schmitt, B. Senger, L. Vidal, J.-C. Voegel, P. Schaaf, Y. Haikel, F. Boulmedais, P. Lavalle, J. Hemmerlé (2015) Cell alignment driven by mechanically induced collagen fiber alignment in collagen/ alginate coatings. Tissue Eng Part C Methods 21: 881-888.

Chen, M.S., A.B. Huber, M.E. van der Haar, M. Frank, L. Schnell, A.A. Spillmann, F. Christ, M.E. Schwab (2000) Nogo-A is a myelin-associated neurite outgrowth inhibitor and an antigen for monoclonal antibody IN-1. Nature 403: 434-439.

Chow, W.N., D.G. Simpson, J.W. Bigbee, R.J. Colello (2007) Evaluating neuronal and glial growth on electrospun polarized matrices: bridging the gap in percussive spinal cord injuries. Neuron Glia Biol 3: 119-126.

Chung, W.S., N.J. Allen, C. Eroglu (2015) Astrocytes control synapse formation, function, and elimination. Cold Spring Harb Perspect Biol 7: a020370.

Chvatal, S.A., Y.T. Kim, A.M. Bratt-Leal, H. Lee, R.V. Bellamkonda (2008) Spatial distribution and acute anti-inflammatory effects of Methylprednisolone after sustained local delivery to the contused spinal cord. Biomaterials 29: 1967-1975.

Clarke, L.E., S.A. Liddelow, C. Chakraborty, A.E. Münch, M. Heiman, B.A. Barres (2018) Normal aging induces A1-like astrocyte reactivity. Proc Natl Acad Sci 115: E1896-E1905.

Cramer, S.C., L. Lastra, M.G. Lacourse, M.J. Cohen (2005) Brain motor system function after chronic, complete spinal cord injury. Brain 128: 2941-2950.

Cregg, J.M., M.A. DePaul, A.R. Filous, B.T. Lang, A. Tran, J. Silver (2014) Functional regeneration beyond the glial scar. Exp Neurol 253: 197-207.

Davies, S.J., D.R. Goucher, C. Doller, J. Silver (1999) Robust regeneration of adult sensory axons in degenerating white matter of the adult rat spinal cord. J Neurosci 1999: 58105822.
De Astis, S., I. Corradini, R. Morini, S. Rodighiero, R. Tomasoni, C. Lenardi, C. Verderio, P. Milani, M. Matteoli (2013) Nanostructured $\mathrm{TiO}_{2}$ surfaces promote polarized activation of microglia, but not astrocytes, toward a proinflammatory profile. Nanoscale 5: 1096310974.

Dela Peña, I., P.R. Sanberg, S. Acosta, N. Tajiri, S.Z. Lin, C.V. Borlongan (2014) Stem cells and G-CSF for treating neuroinflammation in traumatic brain injury: aging as a comorbidity factor. J Neurosurg Sci 58: 145-149.

DeVivo, M.J., B.K. Go, A.B. Jackson (2002) Overview of the National Spinal Cord Injury Statistical Center database. J Spinal Cord Med 25: 335-338.

Donnelly, D.J., P.G. Popovich (2008) Inflammation and its role in neuroprotection, axonal regeneration and functional recovery after spinal cord injury. Exp Neurol 209: 378-388.

Dumont, C.M., D.J. Margul, L.D. Shea (2016) Tissue engineering approaches to modulate the inflammatory milieu following spinal cord injury. Cells Tissues Organs 202: 52-66.

Dyck, S.M., S. Karimi-Abdolrezaee (2015) Chondroitin sulfate proteoglycans: key modulators in the developing and pathologic central nervous system. Exp Neurol 269: 169-187.

East, E., J.P. Golding, J.B. Phillips (2009) A versatile $3 \mathrm{D}$ culture model facilitates monitoring of astrocytes undergoing reactive gliosis. J Tissue Eng Regen Med 3: 634-646.

East, E., N. Johns, M. Georgiou, J.P. Golding, A.J. Loughlin, P.J. Kingham, J.B. Phillips (2013) A $3 \mathrm{D}$ in vitro model reveals differences in the astrocyte response elicited by potential stem cell therapies for CNS injury. Regen Med 8: 739-746.

Eddleston, M., L. Mucke (1993) Molecular profile of reactive astrocytes-Implications for their role in neurologic disease. Neuroscience 54: $15-36$.

Endoh, M., K. Maiese, J. Wagner (1994) Expression of the inducible form of nitric oxide synthase by reactive astrocytes after transient global ischemia. Brain Res 651: 92-100.

Eng, L.F. (1985) Glial fibrillary acidic protein (GFAP): the major protein of glial intermediate filaments in differentiated astrocytes. J Neuroimmunol 8: 203-214.

Eng, L.F., R.S. Ghirnikar, Y.L. Lee (2000) Glial fibrillary acidic protein: GFAP-thirty-one years (1969-2000). Neurochem Res 25: 1439-1451.

Ereifej, E.S., H.W. Matthew, G. Newaz, A. Mukhopadhyay, G. Auner, I. Salakhutdinov, P.J. Vandevord (2013) Nanopatterning effects on astrocyte reactivity. J Biomed Mater Res A 101: 1743-1757.

Farooque, M., L. Hillered, A. Holtz, Y. Olsson (1996) Changes of extracellular levels of amino acids after graded compression trauma to the spinal cord: an experimental study in the rat using microdialysis. J Neurotrauma 13: 537-548.

Faulkner, J.R. (2004) Reactive astrocytes protect tissue and preserve function after spinal cord injury. J Neurosci 24: 2143-2155.
Frampton, J.P., M.R. Hynd, M.L. Shuler, W. Shain (2011) Fabrication and optimization of alginate hydrogel constructs for use in $3 \mathrm{D}$ neural cell culture. Biomed Mater 6: 15002-15002.

Ganguly, D., C.D.L. Johnson, M.K. Gottipati, D. Rende, D.A. Borca-Tasciuc, R.J. Gilbert (2018) Specific nanoporous geometries on anodized alumina surfaces influence astrocyte adhesion and glial fibrillary acidic protein immunoreactivity levels. ACS Biomater Sci Eng 4: 128-141.

Gelain, F., S. Panseri, S. Antonini, C. Cunha, M. Donega, J. Lowery, F. Taraballi, G. Cerri, M. Montagna, F. Baldissera, A. Vescovi (2011) Transplantation of nanostructured composite scaffolds results in the regeneration of chronically injured spinal cords. ACS Nano 5: 227-236

Georges, P.C., W.J. Miller, D.F. Meaney, E.S. Sawyer, P.A. Janmey (2006) Matrices with compliance comparable to that of brain tissue select neuronal over glial growth in mixed cortical cultures. Biophys J 90: 3012-3018.

Gerndt, S.J., J.L. Rodriguez, J.W. Pawlik, P.A. Taheri, W.L. Wahl, A.J. Micheals, S.M. Papadopoulos (1997) Consequences of high-dose steroid therapy for acute spinal cord injury. J Trauma 42: 279-284.

Giannetti, S., L. Lauretti, E. Fernandez, F. Salvinelli, G. Tamburrini, R. Pallini (2001) Acrylic hydrogel implants after spinal cord lesion in the adult rat. Neurol Res 23: 405-409.

Gilbert, R.J., C.J. Rivet, J.M. Zuidema, P.G. Popovich (2011) Biomaterial design considerations for repairing the injured spinal cord. Crit Rev Biomed Eng 39: 125-180.

Gilletti, A., J. Muthuswamy (2006) Brain micromotion around implants in the rodent somatosensory cortex. J Neural Eng 3: 189-195.

Gottipati, M.K., E. Bekyarova, M. Brenner, R.C. Haddon, V. Parpura (2014) Changes in the morphology and proliferation of astrocytes induced by two modalities of chemically functionalized single-walled carbon nanotubes are differentially mediated by glial fibrillary acidic protein. Nano Lett 14: 3720 3727.

Gottipati, M.K., J.J. Samuelson, I. Kalinina, E. Bekyarova, R.C. Haddon, V. Parpura (2013) Chemically functionalized single-walled carbon nanotube films modulate the morphofunctional and proliferative characteristics of astrocytes. Nano Lett 13: 4387-4392.

Goursaud, S., E.N. Kozlova, J.M. Maloteaux, E. Hermans (2009) Cultured astrocytes derived from corpus callosum or cortical grey matter show distinct glutamate handling properties. J Neurochem 108: 1442-1452.

Goyal, R., L.K. Macri, H.M. Kaplan, J. Kohn (2016) Nanoparticles and nanofibers for topical drug delivery. J Control Release 240: 7792.

Grimpe, B. (2004) A novel DNA enzyme reduces glycosaminoglycan chains in the glial scar and allows microtransplanted dorsal root ganglia axons to regenerate beyond lesions in the spinal cord. J Neurosci 24: 1393-1397. 
Haggerty, A.E., I. Maldonado-Lasunción, M. Oudega (2018) Biomaterials for revascularization and immunomodulation after spinal cord injury. Biomed Mater 13: 044105.

Haggerty, A.E., M. Oudega (2013) Biomaterials for spinal cord repair. Neurosci Bull 29: 445459.

Hall, E.D. (2011) Antioxidant therapies for acute spinal cord injury. Neurotherapeutics 8: 152167.

Hall, E.D., J.M. Braughler (1982) Effects of intravenous methylprednisolone on spinal cord lipid peroxidation and $(\mathrm{Na}+, \mathrm{K}+)$-ATPase activity: dose-response analysis during 1st hour after contusion injury in the cat. J Neurosurg 57: 247-253.

Hansen, D.B., N. Garrido-Comas, M. Salter, R. Fern (2015) HCO-3-independent $\mathrm{pH}$ regulation in astrocytes in situ is dominated by $\mathrm{V}$ ATPase. J Biol Chem 290: 8039-8047.

Hara, M., K. Kobayakawa, Y. Ohkawa, H. Kumamaru, K. Yokota, T. Saito, K. Kijima, S. Yoshizaki, K. Harimaya, Y. Nakashima, S. Okada (2017) Interaction of reactive astrocytes with type I collagen induces astrocytic scar formation through the integrin- $\mathrm{N}$-cadherin pathway after spinal cord injury. Nat Med 23: 818828 .

Hawkins, B.T., S. Grego, K.L. Sellgren (2015) Three-dimensional culture conditions differentially affect astrocyte modulation of brain endothelial barrier function in response to transforming growth factor $\beta 1$. Brain Res 1608: 167-176.

Hernandez, M.R. (2000) The optic nerve head in glaucoma: role of astrocytes in tissue remodeling. Prog Retin Eye Res 19: 297-321.

Herrmann, J.E., T. Imura, B. Song, J. Qi, Y. Ao, T.K. Nguyen, R.A. Korsak, K. Takeda, S. Akira, M.V. Sofroniew (2008) STAT3 is a critical regulator of astrogliosis and scar formation after spinal cord injury. J Neurosci 28: 72317243.

Hewett, J.A. (2009) Determinants of regional and local diversity within the astroglial lineage of the normal central nervous system. J Neurochem 110: 1717-1736.

Hoare, T.R., D.S. Kohane (2008) Hydrogels in drug delivery: progress and challenges. Polymer 49: 1993-2007.

Hol, E.M., M. Pekny (2015) Glial fibrillary acidic protein (GFAP) and the astrocyte intermediate filament system in diseases of the central nervous system. Curr Opin Cell Biol 32: 121130.

Horn, K.P., S.A. Busch, A.L. Hawthorne, N. van Rooijen, J. Silver (2008) Another barrier to regeneration in the CNS: activated macrophages induce extensive retraction of dystrophic axons through direct physical interactions. J Neurosci 28: 9330-9341.

Hsiao, T.W., V.P. Swarup, B. Kuberan, P.A. Tresco, V. Hlady (2013) Astrocytes specifically remove surface-adsorbed fibrinogen and locally express chondroitin sulfate proteoglycans. Acta Biomater 9: 7200-7208.
Hsiao, T.W., P.A. Tresco, V. Hlady (2015) Astrocytes alignment and reactivity on collagen hydrogels patterned with ECM proteins. Biomaterials 39: $124-130$

Hsiao, T.W., P.A. Tresco, V. Hlady (2018) Astrocyte spreading and migration on aggrecanlaminin dot gradients. Biointerphases 13: 01A401.

Hu, W.W., Z. Wang, S.S. Zhang, L. Jiang, J. Zhang, X. Zhang, Q.F. Lei, H.J. Park, W.J. Fang, Z. Chen (2014) Morphology and functions of astrocytes cultured on water-repellent fractal tripalmitin surfaces. Biomaterials 35: 73867397.

Hurtado, A., J.M. Cregg, H.B. Wang, D.F. Wendell, M. Oudega, R.J. Gilbert, J.W. McDonald (2011) Robust CNS regeneration after complete spinal cord transection using aligned poly-L-lactic acid microfibers. Biomaterials 32: 6068-6079.

Hyysalo, A., M. Ristola, T. Joki, M. Honkanen, M. Vippola, S. Narkilahti (2017) Aligned poly( $\varepsilon$ caprolactone) nanofibers guide the orientation and migration of human pluripotent stem cell-derived neurons, astrocytes, and oligodendrocyte precursor cells in vitro. Macromol Biosci 17: 1600517.

Ingavle, G.C., J.K. Leach (2014) Advancements in electrospinning of polymeric nanofibrous scaffolds for tissue engineering. Tissue Eng Part B Rev 20: 277-293.

Jain, A., Y.T. Kim, R.J. McKeon, R.V. Bellamkon$\mathrm{da}$ (2006) In situ gelling hydrogels for conformal repair of spinal cord defects, and local delivery of BDNF after spinal cord injury. Biomaterials 27: 497-504.

Jimenez Hamann, M.C., C.H. Tator, M.S. Shoichet (2005) Injectable intrathecal delivery system for localized administration of EGF and FGF-2 to the injured rat spinal cord. Exp Neurol 194: 106-119.

Jin, X., T. Yamashita (2016) Microglia in central nervous system repair after injury. J Biochem 159: 491-496.

Johnson, P.J., S.R. Parker, S.E. Sakiyama-Elbert (2010) Fibrin-based tissue engineering scaffolds enhance neural fiber sprouting and delay the accumulation of reactive astrocytes at the lesion in a subacute model of spinal cord injury. J Biomed Mater Res A 92: 152-163.

Johnson-Green, P.C., K.E. Dow, R.J. Riopelle (1991) Characterization of glycosaminoglycans produced by primary astrocytes in vitro. Glia 4: 314-321.

Joosten, E.A.J., P.R. Bär, W.H. Gispen (1995) Collagen implants and cortico-spinal axonal growth after mid-thoracic spinal cord lesion in the adult rat. J Neurosci Res 41: 481-490.

Jordan, M.D., B.J. Raos, A.S. Bunting, A.F. Murray, E.S. Graham, C.P. Unsworth (2016) Human astrocytic grid networks patterned in parylene- $\mathrm{C}$ inlayed $\mathrm{SiO}_{2}$ trenches. Biomaterials 105: $117-126$
Kang, Y.M., D.H. Hwang, B.G. Kim, D.H. Go, K.D. Park (2010) Thermosensitive polymerbased hydrogel mixed with the anti-inflammatory agent minocycline induces axonal regeneration in hemisected spinal cord. Macromol Res 18: 399-403.

Katoh-Semba, R., M. Matsuda, K. Kato, A. Oohira (1995) Chondroitin sulphate proteoglycans in the rat brain: candidates for axon barriers of sensory neurons and the possible modification by laminin of their actions. Eur J Neurosci 7: 613-621.

Khaing, Z.Z., B.D. Milman, J.E. Vanscoy, S.K. Seidlits, R.J. Grill, C.E. Schmidt (2011) High molecular weight hyaluronic acid limits astrocyte activation and scar formation after spinal cord injury. J Neural Eng 8: 046033.

Kim, S.H., C. Shin, S.K. Min, S.M. Jung, H.S. Shin (2012) In vitro evaluation of the effects of electrospun PCL nanofiber mats containing the microalgae Spirulina (Arthrospira) extract on primary astrocytes. Colloids Surf B Biointerfaces 90: 113-118.

Kim, Y.T., J.M. Caldwell, R.V. Bellamkonda (2009) Nanoparticle-mediated local delivery of methylprednisolone after spinal cord injury. Biomaterials 30: 2582-2590.

Kimelberg, H.K., M. Nedergaard (2010) Functions of astrocytes and their potential as therapeutic targets. Neurotherapeutics 7: 338353

Klouda, L., A.G. Mikos (2008) Thermoresponsive hydrogels in biomedical applications. Eur J Pharm Biopharm 68: 34-45.

Kretlow, J.D., L. Klouda, A.G. Mikos (2007) Injectable matrices and scaffolds for drug delivery in tissue engineering. Adv Drug Deliv Rev 59: 263-273.

Lanza, R., R. Langer, J.P. Vacanti (2011) Principles of Tissue Engineering. Cambridge, Academic Press.

Lau, C.L., M. Kovacevic, T.S. Tingleff, J.S. Forsythe, H.S. Cate, D. Merlo, C. Cederfur, F.L. Maclean, C.L. Parish, M.K. Horne, D.R. Nisbet, P.M. Beart (2014) 3D electrospun scaffolds promote a cytotrophic phenotype of cultured primary astrocytes. J Neurochem 130: 215-226

Lee, H., R.J. McKeon, R.V. Bellamkonda (2010) Sustained delivery of thermostabilized chABC enhances axonal sprouting and functional recovery after spinal cord injury. Proc Natl Acad Sci USA 107: 3340-3345.

Lee, I.H., E. Lindqvist, O. Kiehn, J. Widenfalk, L. Olson (2005) Glial and neuronal connexin expression patterns in the rat spinal cord during development and following injury. J Comp Neurol 489: 1-10.

Legos, J.J., K.R. Gritman, R.F. Tuma, W.F. Young, C.J. Hodge, M.R. Bullock, R.G. Fessler (2001) Coadministration of methylprednisolone with hypertonic saline solution improves overall neurological function and survival rates in a chronic model of spinal cord injury. Neurosurgery 49: 1427-1433. 
Liddelow, S.A., B.A. Barres (2016) Regeneration: not everything is scary about a glial scar. Nature 532: 182-183.

Liddelow, S.A., B.A. Barres (2017) Reactive astrocytes: production, function, and therapeutic potential. Immunity 46: 957-967.

Liddelow, S.A., K.A. Guttenplan, L.E. Clarke, F.C Bennett, C.J. Bohlen, L. Schirmer, M.L. Bennett, A.E. Münch, W.S. Chung, T.C. Peterson, D.K. Wilton, A. Frouin, B.A. Napier, N. Panicker, M. Kumar, M.S. Buckwalter, D.H. Rowitch, V.L. Dawson, T.M. Dawson, B. Stevens, B.A. Barres (2017) Neurotoxic reactive astrocytes are induced by activated microglia. $\mathrm{Na}$ ture 541: 481-487.

Liu, T., J.D. Houle, J. Xu, B.P. Chan, S.Y. Chew (2012) Nanofibrous collagen nerve conduits for spinal cord repair. Tissue Eng Part A 18: 1057-1066.

Lozano, K. (2000) Vapor-grown carbon-fiber composites: processing and electrostatic dissipative applications. JOM 52: 34-36.

Lundgaard, I., M.J. Osório, B.T. Kress, S. Sanggaard, M. Nedergaard (2014) White matter astrocytes in health and disease. Neuroscience 276: 161-173.

Mabon, P.J., L.C. Weaver, G.A. Dekaban (2000) Inhibition of monocyte/macrophage migration to a spinal cord injury site by an antibody to the integrin $\alpha \mathrm{D}$ : a potential new anti-inflammatory treatment. Exp Neurol 166: $52-$ 64.

Macaya, D.J., K. Hayakawa, K. Arai, M. Spector (2013) Astrocyte infiltration into injectable collagen-based hydrogels containing FGF-2 to treat spinal cord injury. Biomaterials 34 : 3602.

Marchand, R., S. Woerly (1990) Transected spinal cords grafted with in situ self-assembled collagen matrices. Neuroscience 36: 45-60.

Marchand, R., S. Woerly, L. Bertrand, N. Valdes (1993) Evaluation of two cross-linked collagen gels implanted in the transected spinal cord. Brain Res Bull 30: 415-422.

Martín-López, E., F.R. Alonso, M. Nieto-Díaz, M Nieto-Sampedro (2012) Chitosan, gelatin and poly(L-Lysine) polyelectrolyte-based scaffolds and films for neural tissue engineering. J Biomater Sci Polym Ed 23: 207-232.

Mattotti, M., Z. Alvarez, J.A. Ortega, J.A. Planell, E. Engel, S. Alcántara (2012) Inducing functional radial glia-like progenitors from cortical astrocyte cultures using micropatterned PMMA. Biomaterials 33: 1759-1770.

Mazzatenta, A., M. Giugliano, S. Campidelli, L. Gambazzi, L. Businaro, H. Markram, M. Prato, L. Ballerini (2007) Interfacing neurons with carbon nanotubes: electrical signal transfer and synaptic stimulation in cultured brain circuits. J Neurosci 27: 6931-6936.

McAdoo, D.J., G.Y. Xu, G. Robak, M.G. Hughes (1999) Changes in amino acid concentrations over time and space around an impact injury and their diffusion through the rat spinal cord. Exp Neurol 159: 538-544.
McKay, C.A., R.D. Pomrenke, J.S. McLane, N.J. Schaub, E.K. Desimone, L.A. Ligon, R.J. Gilbert (2014) An injectable, calcium responsive composite hydrogel for the treatment of acute spinal cord injury. ACS Appl Mater Interfaces 6: 1424-1438.

McKenzie, J.L., M.C. Waid, R. Shi, T.J. Webster (2004) Decreased functions of astrocytes on carbon nanofiber materials. Biomaterials 25 : 1309-1317.

McKerracher, L., S. David, D.L. Jackson, V. Kottis, R.J. Dunn, P.E. Braun (1994) Identification of myelin-associated glycoprotein as a major myelin-derived inhibitor of neurite growth. Neuron 13: 805-811.

Means, E.D., D.K. Anderson (1983) Neuronophagia by leukocytes in experimental spinal cord injury. J Neuropathol Exp Neurol 42: 707-719.

Meng, F., V. Hlady, P.A. Tresco (2012) Inducing alignment in astrocyte tissue constructs by surface ligands patterned on biomaterials. Biomaterials 33: 1323-1335.

Miller, R.H., M.C. Raff (1984) Fibrous and protoplasmic astrocytes are distinct classes of glial cells. J Neurosci 4: 585-592.

Min, S.K., S.M. Jung, J.H. Ju, Y.S. Kwon, G.H. Yoon, H.S. Shin (2015) Regulation of astrocyte activity via control over stiffness of cellulose acetate electrospun nanofiber. In Vitro Cell Dev Biol Anim 51: 933-940.

Min, S.K., C.R. Kim, S.H. Kim, H.S. Shin (2014) Assessment of morphology, activity, and infiltration of astrocytes on marine EPS-imbedded electrospun PCL nanofiber. J Nanomater 2014: 1-8.

Min, S.K., S.H. Kim, C.R. Kim, S.M. Paik, S.M Jung, H.S. Shin (2013) Effect of topography of an electrospun nanofiber on modulation of activity of primary rat astrocytes. Neurosci Lett 534: 80-84.

Minev, I.R., P. Moshayedi, J.W. Fawcett, S.P. Lacour (2013) Interaction of glia with a compliant, microstructured silicone surface. Acta Biomater 9: 6936-6942.

Mulvihill, J.J.E., J. Raykin, E.J. Snider, L.A. Schildmeyer, I. Zaman, M.O. Platt, D.J. Kelly, C.R. Ethier (2018) Development of a platform for studying $3 \mathrm{D}$ astrocyte mechanobiology: compression of astrocytes in collagen gels. Ann Biomed Eng 46: 365-374.

Nagano, N., M. Aoyagi, K. Hirakawa (1993) Extracellular matrix modulates the proliferation of rat astrocytes in serum-free culture. Glia 8: $71-76$.

Nagy, J.I., J.E. Rash (2000) Connexins and gap junctions of astrocytes and oligodendrocytes in the CNS. Brain Res Rev 32: 29-44.

Namba, R.M., A.A. Cole, K.B. Bjugstad, M.J. Mahoney (2009) Development of porous PEG hydrogels that enable efficient, uniform cellseeding and permit early neural process extension. Acta Biomater 5: 1884-1897.

Nesic, O., J. Lee, Z. Ye, G.C. Unabia, D. Rafati, C.E. Hulsebosch, J.R. Perez-Polo (2006) Acute and chronic changes in aquaporin 4 expression after spinal cord injury. Neuroscience 143: 779-792.
Ni, Y., H. Hu, E.B. Malarkey, B. Zhao, V. Montana, R.C. Haddon, V. Parpura (2005) Chemically functionalized water soluble singlewalled carbon nanotubes modulate neurite outgrowth. J Nanosci Nanotechnol 5: 17071712.

Oberheim, N.A., S.A. Goldman, M. Nedergaard (2012) Heterogeneity of astrocytic form and function. Methods Mol Biol 814: 23-45.

Ochalski, P.A.Y., U.N. Frankenstein, E.L. Hertzberg, J.I. Nagy (1996) Connexin-43 in rat spinal cord: localization in astrocytes and identification of heterotypic astro-oligodendrocytic gap junctions. Neuroscience 76: 931-945.

Okada, S., M. Nakamura, H. Katoh, T. Miyao, T. Shimazaki, K. Ishii, J. Yamane, A. Yoshimura, Y. Iwamoto, Y. Toyama, H. Okano (2006) Conditional ablation of Stat 3 or Socs 3 discloses a dual role for reactive astrocytes after spinal cord injury. Nat Med 12: 829-834.

Olsen, M.L., S.C. Campbell, M.B. McFerrin, C.L Floyd, H. Sontheimer (2010) Spinal cord injury causes a wide-spread, persistent loss of Kir4.1 and glutamate transporter 1: benefit of 17 $\beta$-oestradiol treatment. Brain 133: 1013 1025 .

Perego, C., C. Vanoni, M. Bossi, S. Massari, H. Basudev, R. Longhi, G. Pietrini (2000) The GLT-1 and GLAST glutamate transporters are expressed on morphologically distinct astrocytes and regulated by neuronal activity in primary hippocampal cocultures. J Neurochem 75: 1076-1084.

Pizzorusso, T., P. Medini, N. Berardi, S. Chierzi, J.W. Fawcett, L. Maffei (2002) Reactivation of ocular dominance plasticity in the adult visual cortex. Science 298: 1248-1251.

Pizzorusso, T., P. Medini, S. Landi, S. Baldini, N. Berardi, L. Maffei (2006) Structural and functional recovery from early monocular deprivation in adult rats. Proc Natl Acad Sci 103: 8517-8522.

Placone, A.L., P.M. McGuiggan, D.E. Bergles, H. Guerrero-Cazares, A. Quiñones-Hinojosa, P.C. Searson (2015) Human astrocytes develop physiological morphology and remain quiescent in a novel 3D matrix. Biomaterials 42: 134-143.

Popovich, P.G., P. Wei, B.T. Stokes (1997) Cellular inflammatory response after spinal cord injury in Sprague-Dawley and Lewis rats. J Comp Neurol 377: 443-464.

Potokar, M., J. Jorgačevski, R. Zorec (2016) Astrocyte aquaporin dynamics in health and disease. Int J Mol Sci 17: 1121-1121.

Puschmann, T.B., C. Zandén, Y. De Pablo, F. Kirchhoff, M. Pekna, J. Liu, M. Pekny (2013) Bioactive 3D cell culture system minimizes cellular stress and maintains the in vivo-like morphological complexity of astroglial cells. Glia 61: 432-440.

Qian, T., X. Guo, A.D. Levi, S. Vanni, R.T. Shebert, M.L. Sipski (2005) High-dose methylprednisolone may cause myopathy in acute spinal cord injury patients. Spinal Cord 43: 199-203. 
Qu, J., D. Wang, H. Wang, Y. Dong, F. Zhang, B. Zuo, H. Zhang (2013) Electrospun silk fibroin nanofibers in different diameters support neurite outgrowth and promote astrocyte migration. J Biomed Mater Res A 101 A: 26672678.

Raos, B.J., C.S. Doyle, M.C. Simpson, E.S. Graham, C.P. Unsworth (2018) Selective PEGylation of parylene- $\mathrm{C} / \mathrm{SiO}_{2}$ substrates for improved astrocyte cell patterning. Sci Rep 8: 2754-2754.

Rathore, K.I., J.L. Berard, A. Redensek, S. Chierzi, R. Lopez-Vales, M. Santos, S. Akira, S. David (2011) Lipocalin 2 plays an immunomodulatory role and has detrimental effects after spinal cord injury. J Neurosci 31: 13412-13419.

Recknor, J.B., J.C. Recknor, D.S. Sakaguchi, S.K. Mallapragada (2004) Oriented astroglial cell growth on micropatterned polystyrene substrates. Biomaterials 25: 2753-2767.

Ren, H., M. Han, J. Zhou, Z.F. Zheng, P. Lu, J.J. Wang, J.Q. Wang, Q.J. Mao, J.Q. Gao, H.W. Ouyang (2014) Repair of spinal cord injury by inhibition of astrocyte growth and inflammatory factor synthesis through local delivery of Flavopiridol in PLGA nanoparticles. Biomaterials 35: 6585-6594.

Ricoult, S.G., J.S. Goldman, D. Stellwagen, D. Juncker, T.E. Kennedy (2012) Generation of microisland cultures using microcontact printing to pattern protein substrates. J Neurosci Methods 208: 10-17.

Rocha, D.N., J.P. Ferraz-Nogueira, C.C. Barrias, J.B. Relvas, A.P. Pêgo (2015) Extracellular environment contribution to astrogliosis - lessons learned from a tissue engineered 3D model of the glial scar. Front Cell Neurosci 9: 377.

Rolls, A., R. Shechter, M. Schwartz (2009) The bright side of the glial scar in CNS repair. Nat Rev Neurosci 10: 235-241.

Roman, J.A., T.L. Niedzielko, R.C. Haddon, V. Parpura, C.L. Floyd (2011) Single-walled carbon nanotubes chemically functionalized with polyethylene glycol promote tissue repair in a rat model of spinal cord injury. J Neurotrauma 28: 2349-2362.

Ross, A.M., Z. Jiang, M. Bastmeyer, J. Lahann (2012) Physical aspects of cell culture substrates: topography, roughness, and elasticity. Small 8: 336-355.

Scemes, E., D.C. Spray (2012) Extracellular K+ and astrocyte signaling via connexin and pannexin channels. Neurochem Res 37: 23102316.

Schaub, N.J., R.J. Gilbert (2011) Controlled release of 6-aminonicotinamide from aligned, electrospun fibers alters astrocyte metabolism and dorsal root ganglia neurite outgrowth. J Neural Eng 8: 46026.

Schaub, N.J., C.D. Johnson, B. Cooper, R.J. Gilbert (2016) Electrospun fibers for spinal cord injury research and regeneration. J Neurotrauma 33: 1405-1415.
Silva, G.A., C. Czeisler, K.L. Niece, E. Beniash, D.A. Harrington, J.A. Kessler, S.I. Stupp (2004) Selective differentiation of neural progenitor cells by high-epitope density nanofibers. Science 303: 1352-1355.

Silva, T.A., H. Zanin, P.W. May, E.J. Corat, O. Fatibello-Filho (2014) Electrochemical performance of porous diamond-like carbon electrodes for sensing hormones, neurotransmitters, and endocrine disruptors. ACS Appl Mater Interfaces 6: 21086-21092.

Silver, J., J.H. Miller (2004) Regeneration beyond the glial scar. Nat Rev Neurosci 5: 146-156.

Singh, A.V., M. Raymond, F. Pace, A. Certo, J.M. Zuidema, C.A. McKay, R.J. Gilbert, X.L. Lu, L.Q. Wan (2015) Astrocytes increase ATP exocytosis mediated calcium signaling in response to microgroove structures. Sci Rep 5: 7847.

Sofroniew, M.V., H.V. Vinters (2010) Astrocytes: biology and pathology. Acta Neuropathol 119: 7-35

Spencer, K.C., J.C. Sy, R. Falcón-Banchs, M.J Cima (2017) A three dimensional in vitro glial scar model to investigate the local strain effects from micromotion around neural implants. Lab Chip 17: 795-804.

Stout, C.E., J.L. Costantin, C.C.G. Naus, A.C. Charles (2002) Intercellular calcium signaling in astrocytes via ATP release through connexin hemichannels. J Biol Chem 277: 1048210488.

Straley, K.S., C.W.P. Foo, S.C. Heilshorn (2010) Biomaterial design strategies for the treatment of spinal cord injuries. J Neurotrauma 27: 1-19.

Struve, J., P.C. Maher, Y.Q. Li, S. Kinney, M.G. Fehlings, C. Kuntz Iv, L.S. Sherman (2005) Disruption of the hyaluronan-based extracellular matrix in spinal cord promotes astrocyte proliferation. Glia 52: 16-24.

Stys, P.K. (1998) Anoxic and ischemic injury of myelinated axons in CNS white matter: from mechanistic concepts to therapeutics. J Cereb Blood Flow Metab 18: 2-25.

Sun, D., M. Lye-Barthel, R.H. Masland, T.C. Jakobs (2010) Structural remodeling of fibrous astrocytes after axonal injury. J Neurosci 30: 14008-14019.

Sun, T., P.S. Donoghue, J.R. Higginson, N. Gadegaard, S.C. Barnett, M.O. Riehle (2011) The interactions of astrocytes and fibroblasts with defined pore structures in static and perfusion cultures. Biomaterials 32: 2021-2031.

Taoka, Y., K. Okajima, M. Uchiba, K. Murakami, S. Kushimoto, M. Johno, M. Naruo, H. Okabe, K. Takatsuki (1997) Role of neutrophils in spinal cord injury in the rat. Neuroscience 79 : 1177-1182.

Tator, C.H. (1991) Review of experimental spinal cord injury with emphasis on the local and systemic circulatory effects. Neurochirurgie 37: 291-302.

Tator, C.H., M.G. Fehlings (1991) Review of the secondary injury theory of acute spinal cord trauma with emphasis on vascular mechanisms. J Neurosurg 75: 15-26.
Taylor, S.J., E.S. Rosenzweig, J.W. McDonald, S.E. Sakiyama-Elbert (2006) Delivery of neurotrophin-3 from fibrin enhances neuronal fiber sprouting after spinal cord injury. J Control Release 113: 226-235.

Tester, N.J., A.H. Plaas, D.R. Howland (2007) Effect of body temperature on chondroitinase $A B C$ 's ability to cleave chondroitin sulfate glycosaminoglycans. J Neurosci Res 85: 11101118.

Totoiu, M.O., H.S. Keirstead (2005) Spinal cord injury is accompanied by chronic progressive demyelination. J Comp Neurol 486: 373-383.

Tsintou, M., K. Dalamagkas, A.M. Seifalian (2015) Advances in regenerative therapies for spinal cord injury: a biomaterials approach. Neural Regen Res 10: 726-742.

Turner, A.M.P., N. Dowell, S.W.P. Turner, L. Kam, M. Isaacson, J.N. Turner, H.G. Craighead, W. Shain (2000) Attachment of astroglial cells to microfabricated pillar arrays of different geometries. J Biomed Mater Res 51: 430-441.

Tutak, U., D.M. Doleys (1996) Intrathecal infusion systems for treatment of chronic low back and leg pain of noncancer origin. South Med J 89: 295-300.

Tysseling-Mattiace, V.M., V. Sahni, K.L. Niece, D. Birch, C. Czeisler, M.G. Fehlings, S.I. Stupp, J.A. Kessler (2008) Self-assembling nanofibers inhibit glial scar formation and promote axon elongation after spinal cord injury. J Neurosci 28: 3814-3823.

Ugbode, C.I., W.D. Hirst, M. Rattray (2016) Astrocytes grown in Alvetex ${ }^{\circledR}$ three dimensional scaffolds retain a non-reactive phenotype. Neurochem Res 41: 1857-1867.

Unsworth, C.P., H. Holloway, E. Delivopoulos, A.F. Murray, M.C. Simpson, M.E. Dickinson, E.S. Graham (2011) Patterning and detailed study of human hNT astrocytes on parylene$\mathrm{C} /$ silicon dioxide substrates to the single cell level. Biomaterials 32: 6541-6550.

Visavadiya, N.P., S.P. Patel, J.L. VanRooyen, P.G. Sullivan, A.G. Rabchevsky (2016) Cellular and subcellular oxidative stress parameters following severe spinal cord injury. Redox Biol 8: 59-67.

Weightman, A.P., M.R. Pickard, Y. Yang, D.M. Chari (2014) An in vitro spinal cord injury model to screen neuroregenerative materials. Biomaterials 35: 3756-3765.

Wilhelmsson, U., E.A. Bushong, D.L. Price, B.L. Smarr, V. Phung, M. Terada, M.H. Ellisman, M. Pekny (2006) Redefining the concept of reactive astrocytes as cells that remain within their unique domains upon reaction to injury. Proc Natl Acad Sci USA 103: 17513-17518.

Willerth, S.M., S.E. Sakiyama-Elbert (2007) Approaches to neural tissue engineering using scaffolds for drug delivery. Adv Drug Deliv Rev 59: 325-338.

Wilson, J.X. (1997) Antioxidant defense of the brain: a role for astrocytes. Can J Physiol Pharmacol 75: 1149-1163. 
Winter, C.C., K.S. Katiyar, N.S. Hernandez, Y.J. Song, L.A. Struzyna, J.P. Harris, D. Kacy Cullen (2016) Transplantable living scaffolds comprised of micro-tissue engineered aligned astrocyte networks to facilitate central nervous system regeneration. Acta Biomater 38: $44-58$.

Woerly, S. (2000) Restorative surgery of the central nervous system by means of tissue engineering using NeuroGel implants. Neurosurg Rev 23: 59-79.

Woerly, S., V.D. Doan, N. Sosa, J. De Vellis, A. Espinosa (2001a) Reconstruction of the transected cat spinal cord following NeuroGel ${ }^{\mathrm{TM}}$ implantation: axonal tracing, immunohistochemical and ultrastructural studies. Int J Dev Neurosci. 19: 63-83.

Woerly, S., V.D. Doan, N. Sosa, J. De Vellis, A. Espinosa-Jeffrey (2004) Prevention of gliotic scar formation by NeuroGel ${ }^{\mathrm{TM}}$ allows partial endogenous repair of transected cat spinal cord. J Neurosci Res 75: 262-272.

Woerly, S., D. Van Doan, F. Evans-Martin, C.G. Paramore, J.D. Peduzzi (2001b) Spinal cord reconstruction using NeuroGel ${ }^{\mathrm{TM}}$ implants and functional recovery after chronic injury. J Neurosci Res 66: 1187-1197.

Xia, M., Y. Zhu (2011) Signaling pathways of ATP-induced PGE2 release in spinal cord astrocytes are EGFR transactivation-dependent. Glia 59: 664-674.
Xia, T., S. Ni, X. Li, J. Yao, H. Qi, X. Fan, J. Wang (2013) Sustained delivery of dbcAMP by poly(propylene carbonate) micron fibers promotes axonal regenerative sprouting and functional recovery after spinal cord hemisection. Brain Res 1538: 41-50.

Xie, S., R. Luttge (2014) Imprint lithography provides topographical nanocues to guide cell growth in primary cortical cell culture. Microelectron Eng 124: 30-36.

Xu, G.Y., D.J. McAdoo, M.G. Hughes, G. Robak, R. De Castro (1998) Considerations in the determination by microdialysis of resting extracellular amino acid concentrations and release upon spinal cord injury. Neuroscience 86: 1011-1021.

Yang, H.Y., N. Lieska, D. Shao, V. Kriho, G.D. Pappas (1994) Proteins of the intermediate filament cytoskeleton as markers for astrocytes and human astrocytomas. Mol Chem Neuropathol 21: 155-176.

Yang, Y., L. De Laporte, C.B. Rives, J.H. Jang, W.C. Lin, K.R. Shull, L.D. Shea (2005) Neurotrophin releasing single and multiple lumen nerve conduits. J Control Rel 104: 433-446.

Yiu, G., Z. He (2006) Glial inhibition of CNS axon regeneration. Nat Rev Neurosci 2006 7: 617627.
Young, W., I. Koreh (1986) Potassium and calcium changes in injured spinal cords. Brain Res 365: 42-53.

Zamanian, J.L., L. Xu, L.C. Foo, N. Nouri, L. Zhou, R.G. Giffard, B.A. Barres (2012) Genomic analysis of reactive astrogliosis. J Neurosci 32: 6391-6410.

Zuidema, J.M., G.P. Desmond, C.J. Rivet, K.R Kearns, D.M. Thompson, R.J. Gilbert (2015) Nebulized solvent ablation of aligned PLLA fibers for the study of neurite response to anisotropic-to-isotropic fiber/film transition (AFFT) boundaries in astrocyte-neuron cocultures. Biomaterials 46: 82-94.

Zuidema, J.M., M.C. Hyzinski-García, K. Van Vlasselaer, N.W. Zaccor, G.E. Plopper, A.A. Mongin, R.J. Gilbert (2014) Enhanced GLT-1 mediated glutamate uptake and migration of primary astrocytes directed by fibronectincoated electrospun poly-L-lactic acid fibers. Biomaterials 35: 1439-1449.

Zuidema, J.M., T. Kumeria, D. Kim, J. Kang, J. Wang, G. Hollett, X. Zhang, D.S. Roberts, N. Chan, C. Dowling, E. Blanco-Suarez, N.J. Allen, M.H. Tuszynski, M.J. Sailor (2018) Oriented nanofibrous polymer scaffolds containing protein-loaded porous silicon generated by spray nebulization. Adv Mater 30: 1706785 . 